\title{
Ethnobotanical Study of Medicinal Plants in Chilga District, Northwestern Ethiopia
}

\author{
Tebkew Mekuanent ${ }^{1 *}$, Asfaw Zebene ${ }^{2}$ and Zewudie Solomon ${ }^{3}$ \\ ${ }^{1}$ University of Gondar P. O. Box. 196, Gondar, Ethiopia \\ ${ }^{2}$ Hawassa University Wondo Genet College of Forestry and Natural Resources, P. O. Box. 128, \\ Shashemene Ethiopia; zebeneasfaw@yahoo.co.uk \\ ${ }^{3}$ Addis Ababa Science and Technology University, Addis Ababa, Ethiopia; zew172@yahoo.com
}

\section{Abstract}

The study was conducted to investigate and document medicinal plants and associated knowledge plants in Chilga district, Northwestern Ethiopia. Questionnaire survey, semi-structured interviews, ranking and transect walk techniques were employed for data collection in midland and lowland agroecologies and in natural forests, riverine forests and farmlands. One hundred one medicinal plants were used to treat 52 ( 41 human and 8 livestock and 3 for both) ailments. Herbs (36\%) and shrubs (30\%) were the most widely used plant growth forms. About $89.8 \%$ of remedies are prepared from roots and leaves. Wealthier groups mentioned significant number of medicinal plants than poorest groups in Quavier Lomiye Kebele. Most medicinal plants (68.48 \% in lowland and $76.3 \%$ in midland agroecology) provide other types of uses in addition to medicinal value. However, these plants are threatened by agricultural expansion, fuel wood and construction and overgrazing. Thus, conservation of these plants and associated knowledge base is very essential.

Keywords: Chilga district, woody species, herbaceous species, medicinal plants

\section{Introduction}

Nearly 100,000 plant species have been regularly used for food, shelter and medicines in the world ${ }^{25}$. However, most of the plant is ignored ${ }^{22,25}$ due to lack of documentation, scientific knowledge and dismination, although these medicinal plants are the pillars to the health care system and livelihood of rural community ${ }^{4,23}$.

Higher medicinal plant diversity is comprised in Ethiopia because of the presence of broad latitudinal ranges ${ }^{4}$. In Northwestern parts of Ethiopia, which is endowed with humid, sub-humid, dry, highland and lowland areas, plenty of medicinal plants are present. However, nowadays the extents of medicinal plant resources are highly degraded.

\footnotetext{
*Author for correspondence
}

Email: mekuannttebkew@yahoo.com
About $80 \%$ of the population in Ethiopia is suffering from the lack of healthcare services ${ }^{4,12}$. Thus, in most cases, rural communities depend on medicinal plants to meet their healthcare needs ${ }^{4}$ due to their easily accessibility and affordability ${ }^{20}$.

Identifying medicinal plants, documenting their uses and assessing the threats create a base for local decision making, applying appropriate management, conduct detail pharmacological analysis and select species for different land uses. However, there is scanty of information on their medicinal uses, ecological distribution and conservation.

Medicinal plants and the associated plant use knowledge are documented in specific locations of Africa $^{23}$ and in some parts of Ethiopia ${ }^{3,6,35}$. Furthermore, 
there are many traditional management practices of medicinal plants like cultural taboos and religious rules. Yet, a comprehensive study and documentation of medicinal plants and their associated knowledge in Chilga District was not conducted. Thus, this study was initiated with the objectives to (i) identify medicinal plants in different land uses, Kebeles (Kebele- the lowest administrative unit next to district in Ethiopia) and agroecologies (ii) determining the role of medicinal plants in household livelihood diversification, (iii) document medicinal plant use knowledge and (iv) identify the factors that threaten medicinal plants.

\section{Materials and Methods}

\subsection{Description of the Study Area}

The study was conducted in Chilga district, North Gondar province of the Amhara Regional State. Chilga district is located $12^{\circ} 55^{\prime \prime} \mathrm{N}$ and $37^{\circ} 06^{\prime \prime} \mathrm{E}$. The district had 41 rural Kebele administrative (KAs) and two town associations ${ }^{7}$.

The altitude of the district ranges from 900 to 2267 meter above sea level (m.a.s.l). It had midland (1500-2267 m.a.s.l) and lowland (900-1500 m.a.s.l) agroecology. About 33\% of the District is midland, while $67 \%$ lowland agro-ecology. There are rivers and streams traversing the District and often serving as sources of water for the population ${ }^{7}$. The major soils of Chilga District are $45 \%$ Cambisols, $40 \%$ Vertisols, $15 \%$, and Nitosols ${ }^{7}$. The vegetation is predominantly composed of different woody and herbaceous species. The natural vegetation of Chilga is mainly composed of various lowland and midland species. The temperature ranges from 11 to $32^{\circ} \mathrm{C}$ and mean annual rainfall is between 995 to $1175 \mathrm{~mm}$. The District had a total population of 241,712 and a total area of $3181 \mathrm{~km}^{2}$. The local people are mainly dependent on subsistence mixed agriculture (crop-livestock production and rearing).

\subsection{Selection of Study Sites and Respondents}

The study was carried out in four KAs of Chilga District from October 8 to December 20 2012. District and Kebele experts and knowledgeable persons were first contacted to have general information. In addition, secondary archived materials were reviewed from district agricultural office to get further information. Based on preliminary information, the socio-demographic and biophysical characteristics of the two agro-ecologies (midland and lowland) are not the same, while Kebeles in same agroecology are fundamentally similar from one another. Thus, based on accessibility for data collection and availability of medicinal plants, two sample KAs from each agroecology and two sample villages from each KA were selected (Table 1).

For this study, key informants (KI) and households were participated for data collection. KIs are defined as knowledgeable persons about medicinal plants as well as local conditions. A simple stratification of households (HHs) was conducted by age ( $\leq 40$ and $>40$ ) and wealth

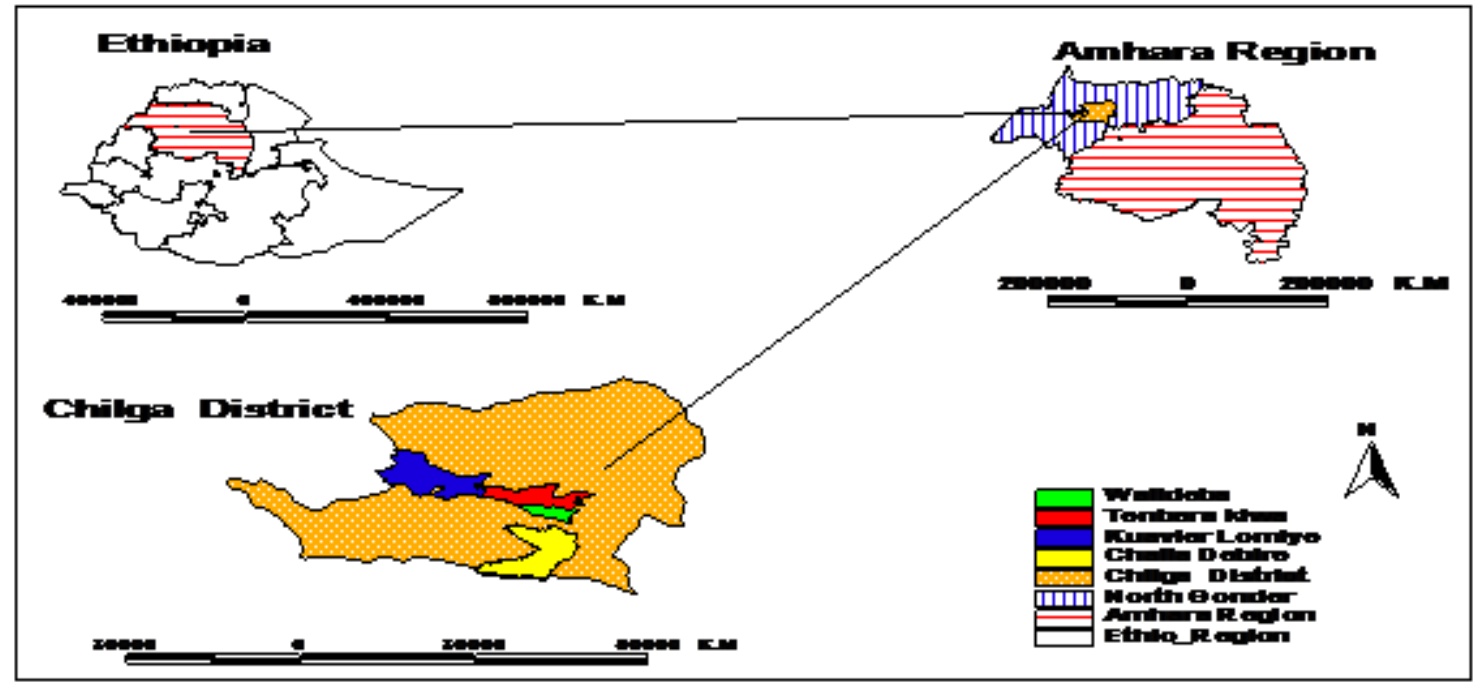

Fig. 1. Location of the study sites in Chilga district, Northwestern Ethiopia. 


\begin{tabular}{lccc} 
Table 1: & \multicolumn{4}{c}{$\begin{array}{c}\text { Sampled Kebeles, villages, households and altitude } \\
\text { in Chilga District, North-western Ethiopia }\end{array}$} \\
\hline Study Kebeles & $\begin{array}{c}\text { Sampled } \\
\text { villages }\end{array}$ & $\begin{array}{c}\text { Number of } \\
\text { Respondents }\end{array}$ & $\begin{array}{c}\text { Altitude } \\
\text { (m.a.s.l) }\end{array}$ \\
\hline Quavier Lomiye & Achera & 12 & Below 1500 \\
& Bele Wuha & 12 & Below 1500 \\
Tenbera Kiwa & Gint & 12 & \\
Walideba & Kete Skangie & 12 & Above 1500 \\
& Mehalgie & 12 & Above 1500 \\
Chalia Debire & Ateraho & 12 & \\
& Awugiber & 12 & \\
\hline
\end{tabular}

Source: CDOA, $2012^{7}$.

(poor, medium and rich), which were commonly used methods in assessing the local knowledge and utilization following the method of Martin ${ }^{26}$. Wealth classification was done by using local wealth classification criteria's in the study KAs. Hence, $12 \mathrm{HHs}$ classified by age category ( $\leq 40$ and $>40$ in 1:1 ratio) and wealth (4 HHs for each wealth class) were taken in each study villages. Thus, 96 $\mathrm{HHs}$ (78 male and 18 female) from the four KA ( $24 \mathrm{HHs}$ from each) were interviewed for the study assuming 5 $\%$ of the population. Out of the total respondents, 24 top ranked KI (all males, three from each village) were selected based on their knowledge.

\subsection{Data Collection}

Questionnaires and checklists were prepared, pre-tested and administered to HHs and KIs, respectively. Consent and a formal permission letter were obtained from district agricultural office. All interviewees were met on a 'one-to-one' basis and were asked the same standard (open- and closed-ended) questions using the local language (Amharic). Information regarding local names of medicinal plants, parts used and diseases treated and application methods were recorded. In addition, information on other uses and threats of medicinal plants were gathered.

Field observations were conducted in areas using transect walk where most of the medicinal plants are grown/cultivated. The purpose of the field observation was to obtain actual information of presence, growth habit, habitat characteristics and identification of medicinal plant species mentioned during the interviews.
A focused group discussion of KI was conducted at each study site to verify the data. All medicinal plants listed in the socio-economic survey were verified and idiosyncratic ideas were removed from the data. Idiosyncratic ideas are ideas/data which represent only the idea of respondent and was not accepted as correct by other key informants. Some ideas of two persons were assumed idiosyncratic and were rejected.

Direct matrix ranking method was exercised for commonly reported multipurpose medicinal plants to assess their relative importance to the local people and the extent of the existing threats related to their use values following the method of Martin ${ }^{26}$. Based on their relative uses, eight most knowledgeable KIs were selected out of the entire KIs and were asked to assign use values for seven medicinal plant species ( $5=$ best, 4 $=$ very good, $3=$ good, $2=$ less used, $1=$ least used and $0=$ not used) to each use category. Frequency of citation as multipurpose species was used in ranking medicinal plant species. Use categories used in the comparison include construction, medicine, fruit/food, fuel wood, shade, farm and household implements, and fence.

Preference ranking was also conducted for 6 commonly reported medicinal plants used to treat rabies in the district following the method of Martin ${ }^{26}$. Rabies was the common ailment for which the local communities do not sought help from primary health services in their locality. Eight KI (most knowledgeable) were selected among $24 \mathrm{KI}$ and participated in the ranking process. The ranking of selected medicinal plants was carried out in pre-designed semi-structured interview items based 
on their personal preference of efficacy from highest score (supposed to be the most effective) to the lowest score (least effective).

The major human and natural factors possibly threatening the survival of medicinal plants were identified through preliminary assessment. Thus, based on the relative importance of the threatening factors, priority ranking was conducted by 8 KIs using the method of Martin ${ }^{26}$. One to five scores were assigned where 1 was for the least and 5 were for the most destructive threat. Then, all ranks were summed up and total ranking was conducted to know the main threats.

All encountered plants were identified and recorded by their vernacular names. Later, converted to their botanical names using flora of Ethiopia and Eritrea ${ }^{9-11,17-}$ ${ }^{19}$ (Edwards 1989), and own experience. Plant specimens were collected and taken to National Herbarium of Addis Ababa University for plant identification.

\subsection{Data Analysis}

Descriptive quantitative and qualitative data analyses were employed after the necessary data collection. Statistical Package for Social Sciences (SPSS) Version16.0 was used for data analysis. The data from ranking methods was presented in the form of ranks. $\mathrm{T}$ test statistical analysis was employed for mean separation. Spearman's Rank Order Correlation was used to test the relation between ages and use knowledge.

\section{Results}

\subsection{Floristic Composition of Medicinal Plants}

One hundred one medicinal plant species belonging to 58 families were recorded in the study area. Among these families, Fabacae was represented by 7 plant species; Myrtaceae, Solanaceae, Apiaceae, Curcurbitaceae, Lamiaceae, Malvaceae and Vitaceae had 5 species each; Euphorbiaceae had 4; Acanthaceae, Aloaceae, Amaranthaceae, Asteraceae, Combretaceae and Polygonaceae 3 plant species each; Asparagaceae, Boraginaceae, Capparidaceae, Phytolaccaceae, Ranunculaceae, Rutaceae, Verbenaceae and Rhammaceae had 2 species each and the remaining other families were represented by one species each (Table 2).

\subsection{Medicinal Plants Habit, Parts Used and Diversity of Uses}

Overall, herbaceous species were the most widely used plants for the treatment of ailments (36\% of species) followed by shrubs (30\%) (Table 3). The remaining $24 \%$ and $11 \%$ of the medicinal species were trees and climbers, respectively. When compared at Kebele level, herbs were the most popularly used plants with proportion of $36 \%$, $35 \%$ and $36 \%$ for Walideba, Chalia Debire and Tenbera, respectively. However, in Quavier Lomiye Kebele shrubs were widely used plants (40.5\%).

Plant remedies were prepared from different parts of the plant (Table 4). About $89.8 \%$ of remedies are prepared from roots and leafs. The remaining $10.2 \%$ are prepared from other plant parts (Stem and root barks, apex, seed, tuber, fruits, sap, oil and fruit coats). In addition, some medicinal plants in the study area were used to treat more than one ailment (Table 2).

Medicinal plants play an important role in provision of other uses such as fuel wood, construction, food, fencing and others in addition to medicinal uses (Table 5). About $68.48 \%$ out of 83 and $76.3 \%$ out of 84 medicinal plants in the lowland and midland agroecology provide additional uses respectively.

\subsection{Preference and Utilization of Medicinal Plants}

Informant consensus on most commonly utilized medicinal plants in the study sites is presented in Table 7. Over all in the study area, Zehneria scabra (mentioned by $31 \%$ informants) and Carisa spinarum (mentioned by $28 \%$ informants) were popularly used medicinal plants. Site-specific preference based on percentage of informants also assured Zehneria scabra was frequently cited medicinal plant in Walideba, Quavier lomiye and Tenbera. Similarly, Carissa spinarum was the most cited plant in Chalia Debire Kebele.

Rabies was the most common disease that the most people visit traditional healer in the study area. Through preference ranking, seven medicinal plants treating rabies were selected (Table 7). Of these, Dorstenia barnimiana and Euphorbia abyssinica were the first and the second ranked species in treating rabies, respectively. However, Cucumis ficifolius was the last ranked species. 


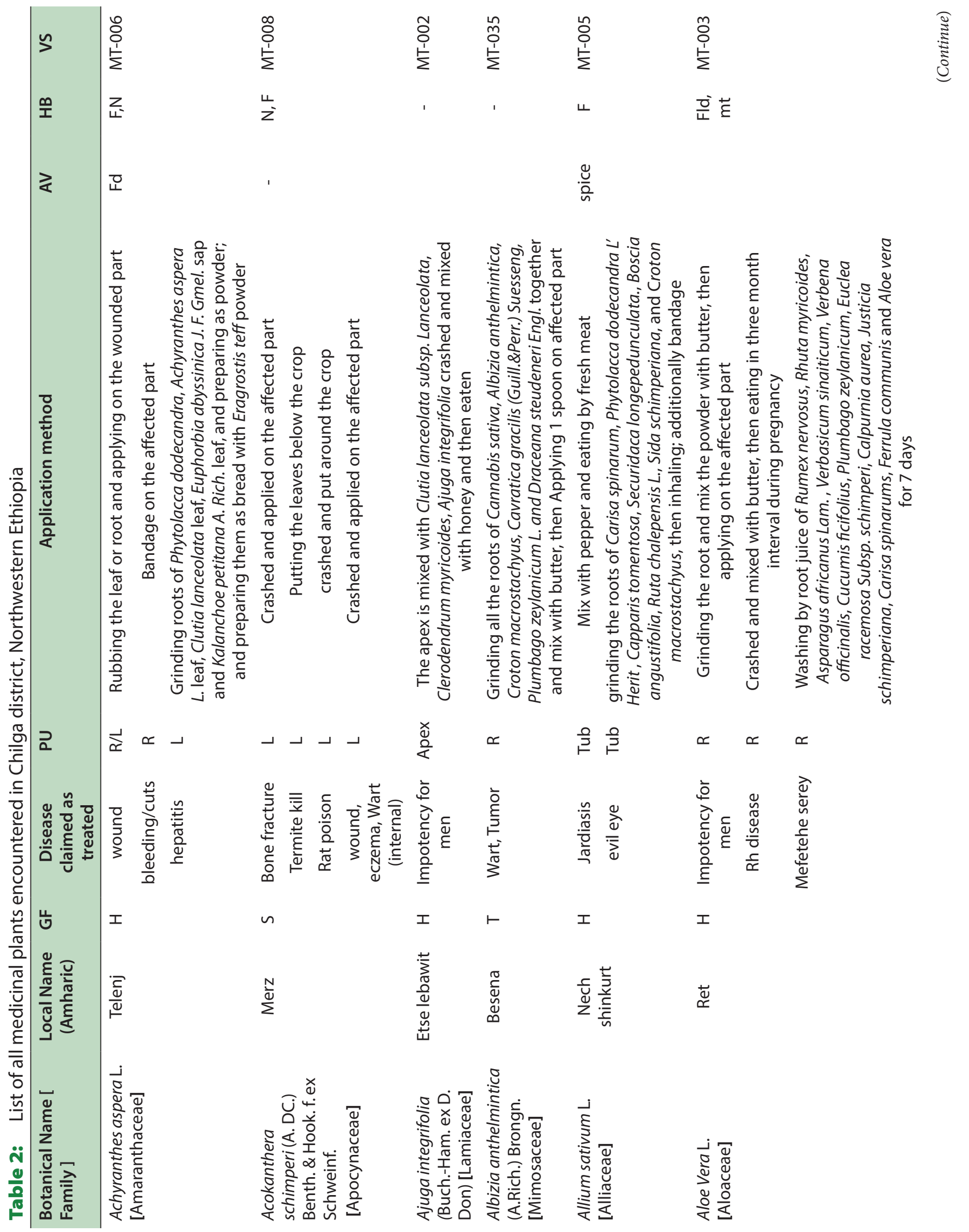




\begin{tabular}{|c|c|c|c|c|}
\hline $\begin{array}{l}\hat{o} \\
\stackrel{1}{2}\end{array}$ & 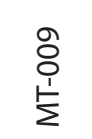 & 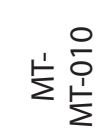 & $\begin{array}{l}\stackrel{n}{0} \\
\stackrel{1}{2}\end{array}$ & 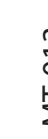 \\
\hline 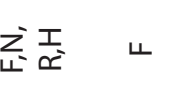 & ธ్ & z & z & \\
\hline 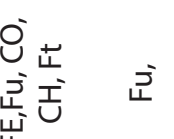 & 岕 & 곤 & 군 & \\
\hline
\end{tabular}
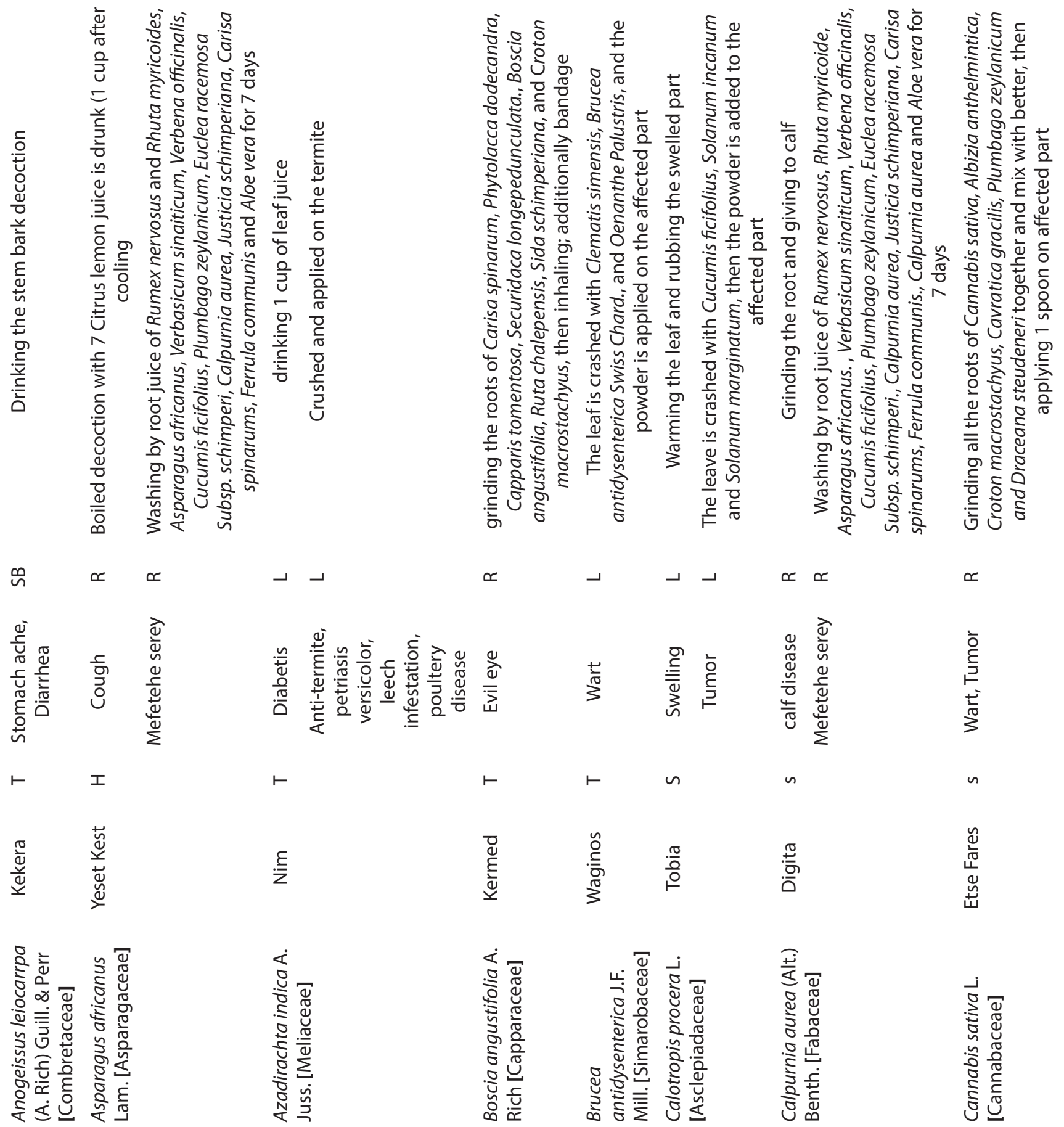


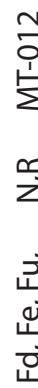

$\begin{array}{ll}\stackrel{0}{0} & \frac{0}{0} \\ \stackrel{1}{1} & \stackrel{!}{\Sigma}\end{array}$

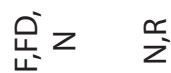

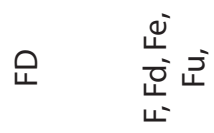

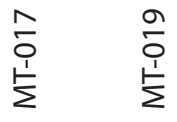

昰

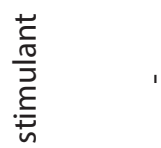

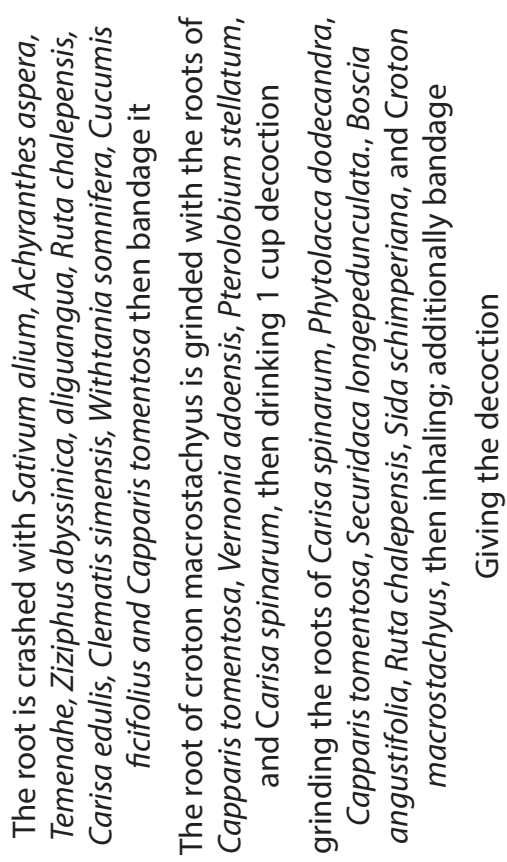

$\propto$

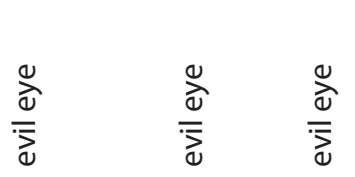

$\backsim$

$\stackrel{\stackrel{\circ}{d}}{\stackrel{5}{5}}$

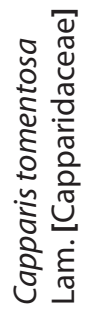

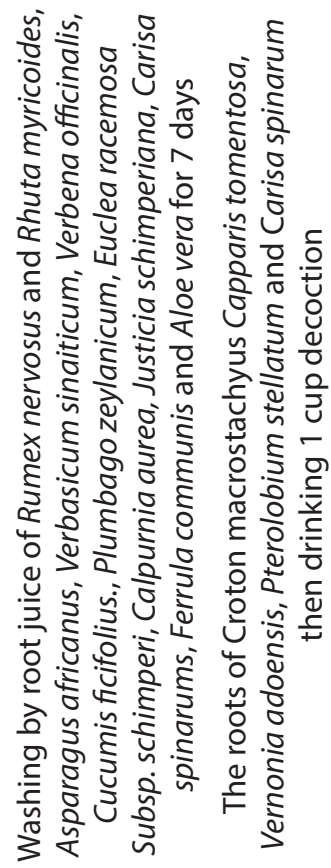
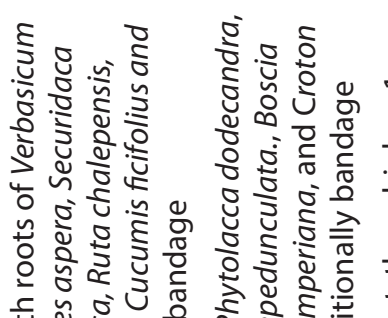

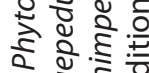

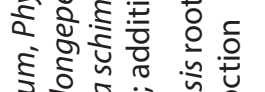

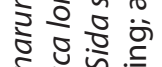

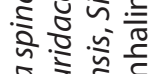

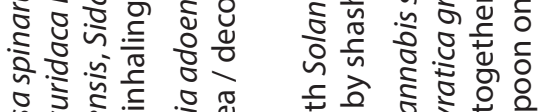
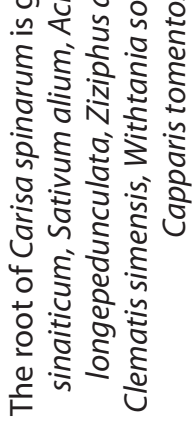

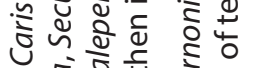
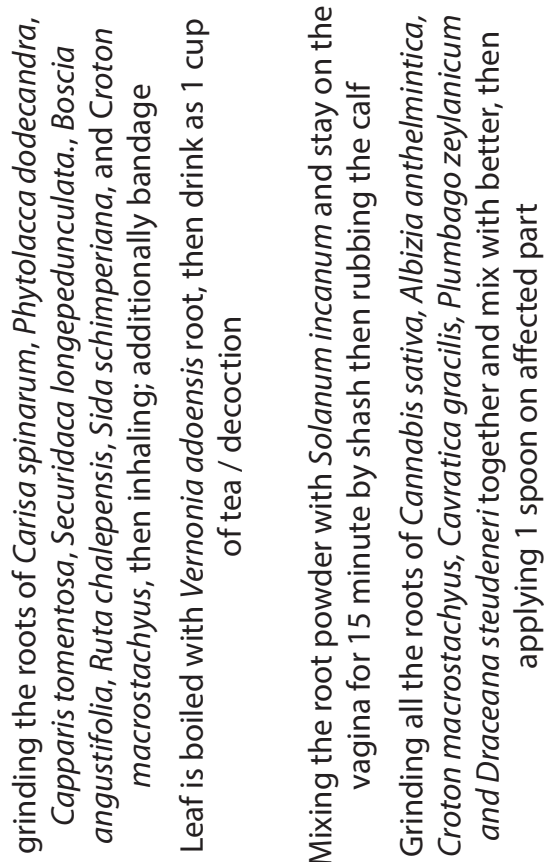

$\propto$

$-\quad \propto \propto$

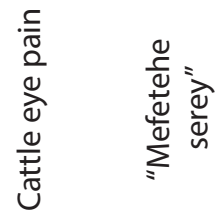

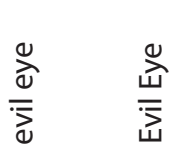

$\frac{\text { ये }}{\sum^{\frac{1}{3}}}$

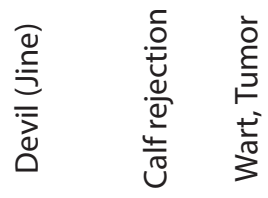

I $\backsim$

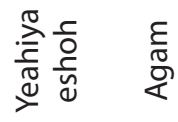

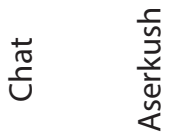

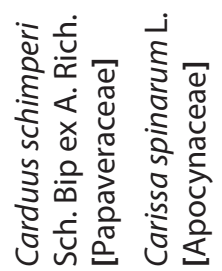

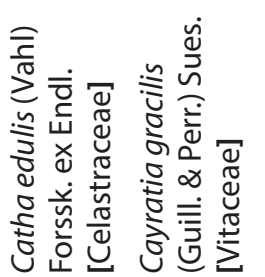




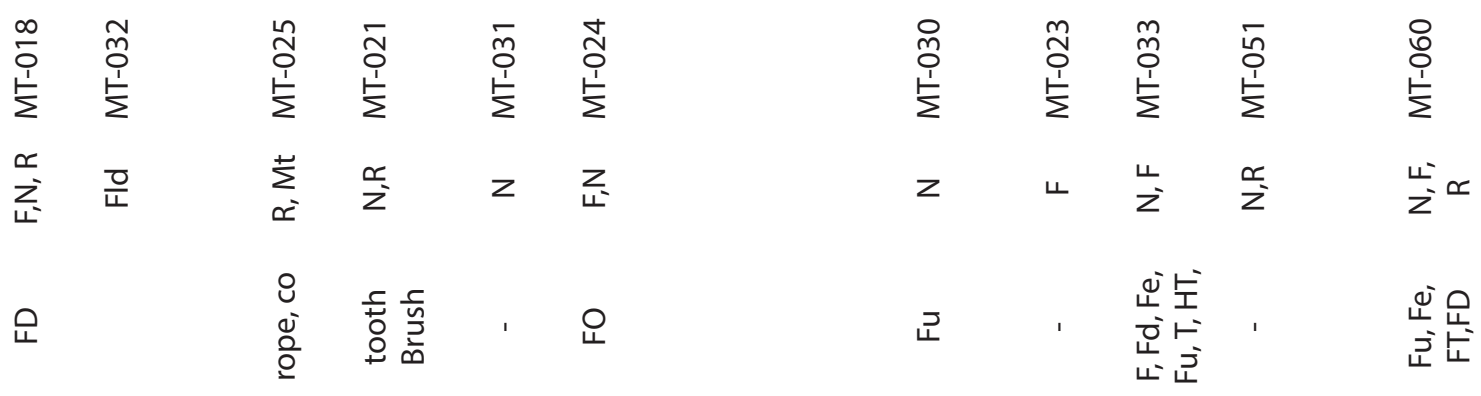
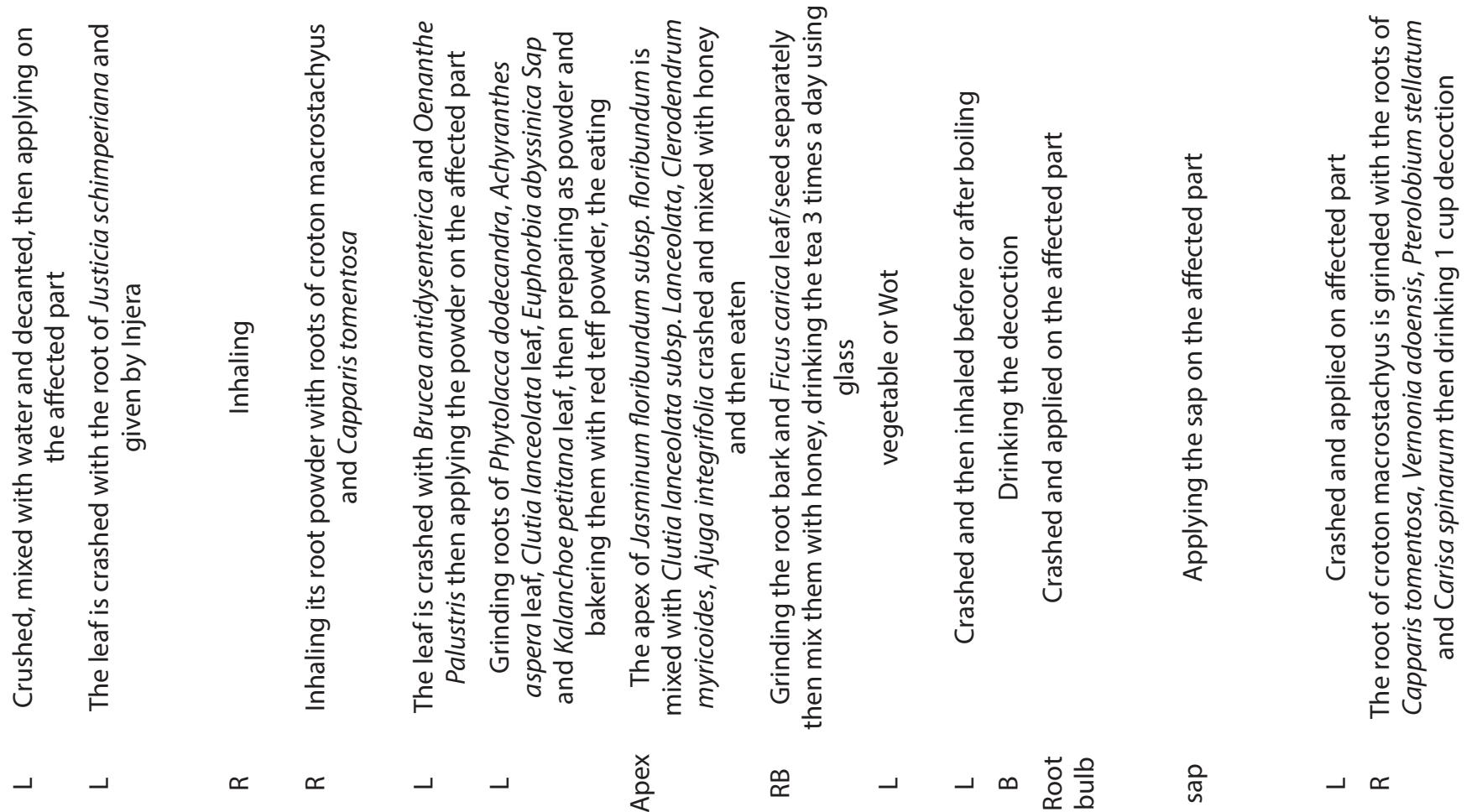

1

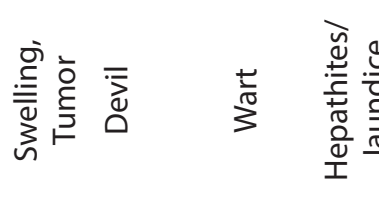

ঐ্ঠ

$\dddot{\Upsilon}$

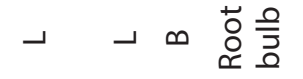

‡

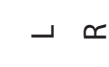

I $I$

$\cup \quad n$

$\cup \quad$ エ

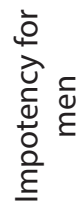

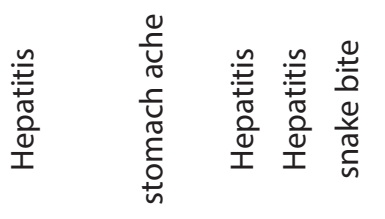

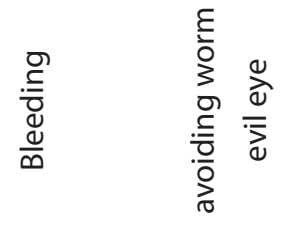

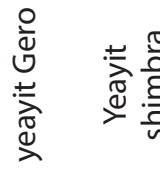

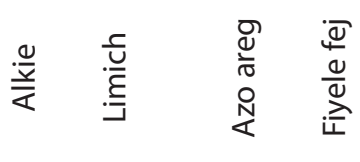

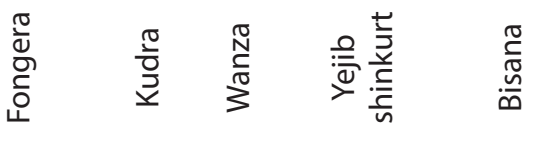
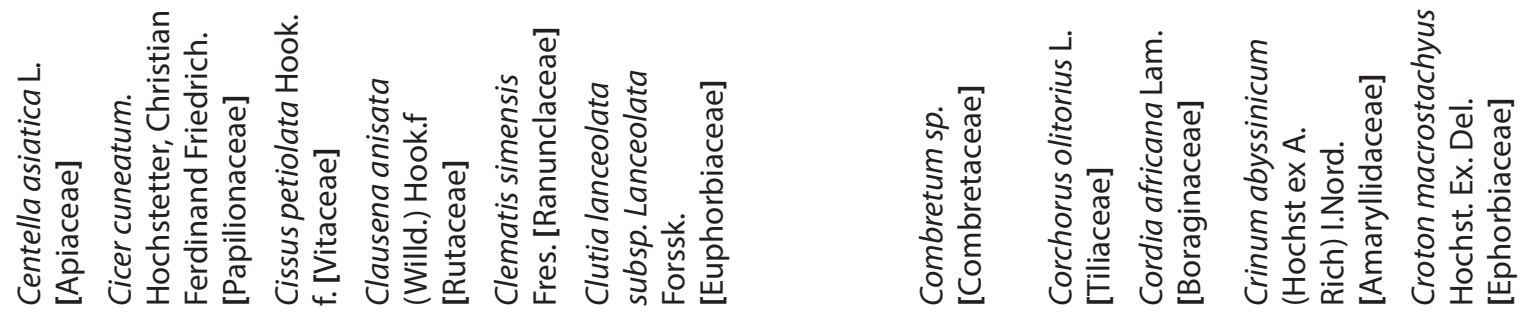
$\bar{\infty}$
o
$\stackrel{1}{\Sigma}$

$\stackrel{\infty}{z}$

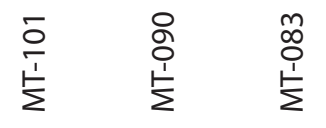

它岂忘采次

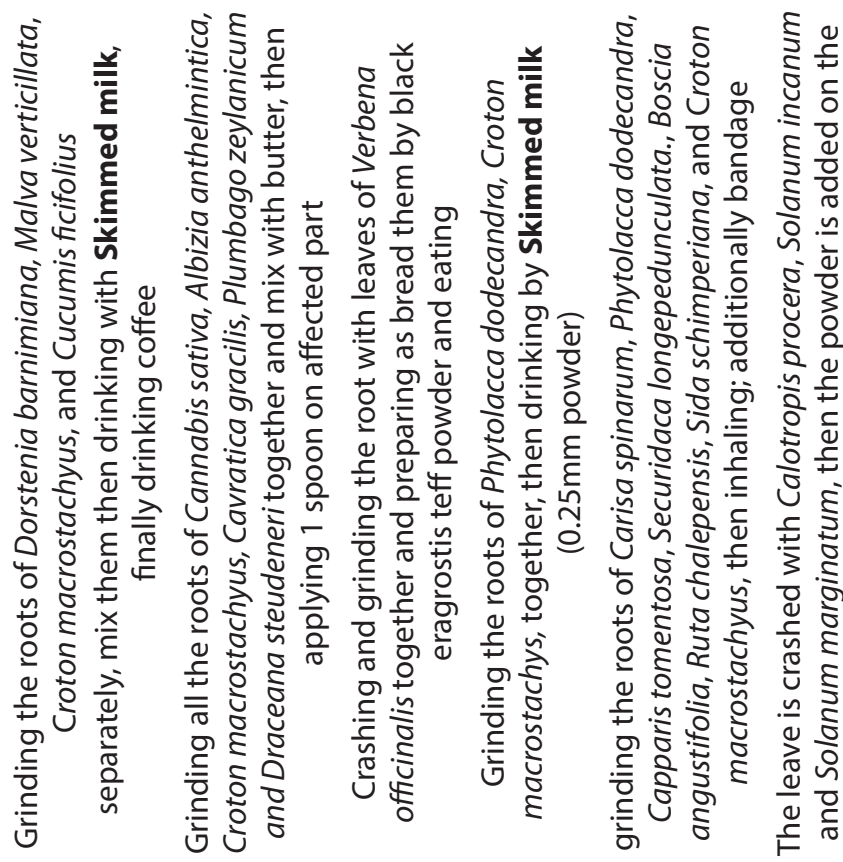

$\simeq$

$\propto$

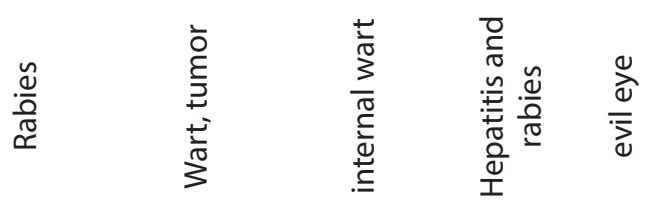

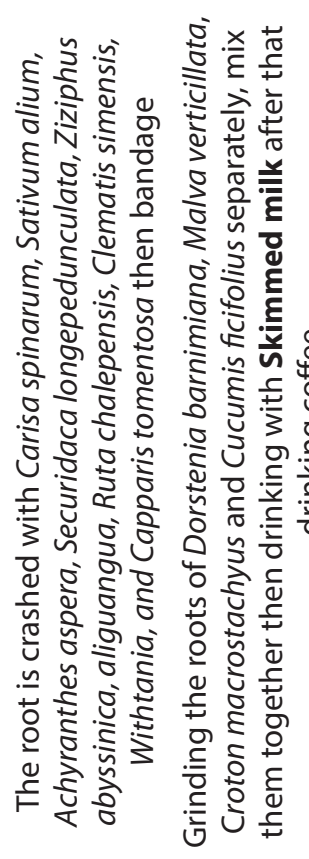

$\simeq$

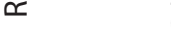

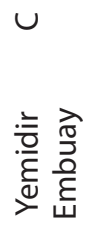

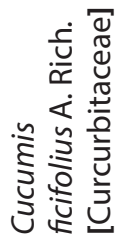

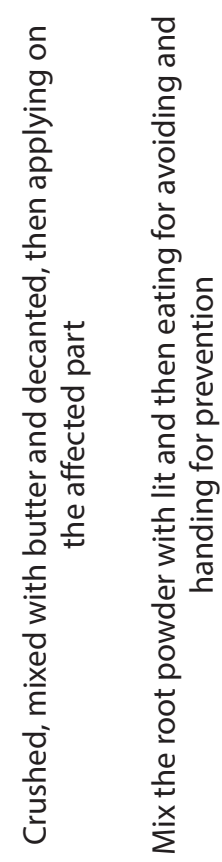

$\triangleright$

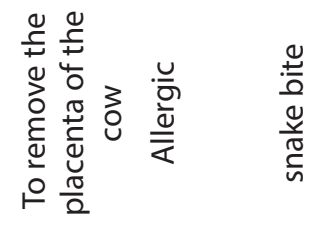

I $I U$

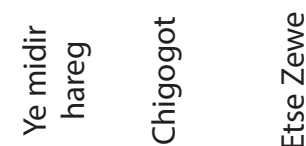

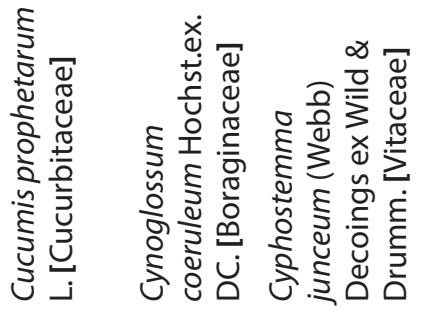




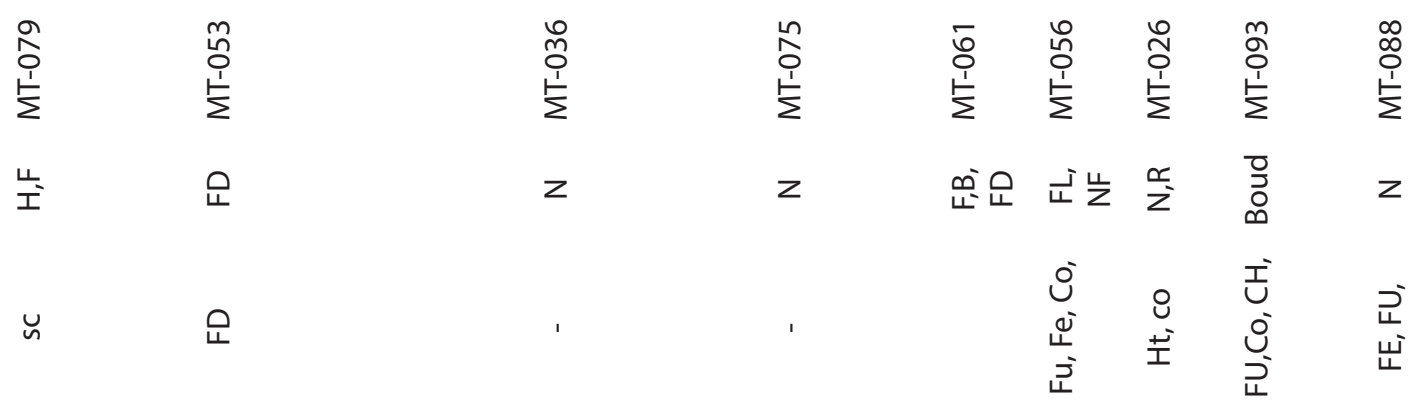
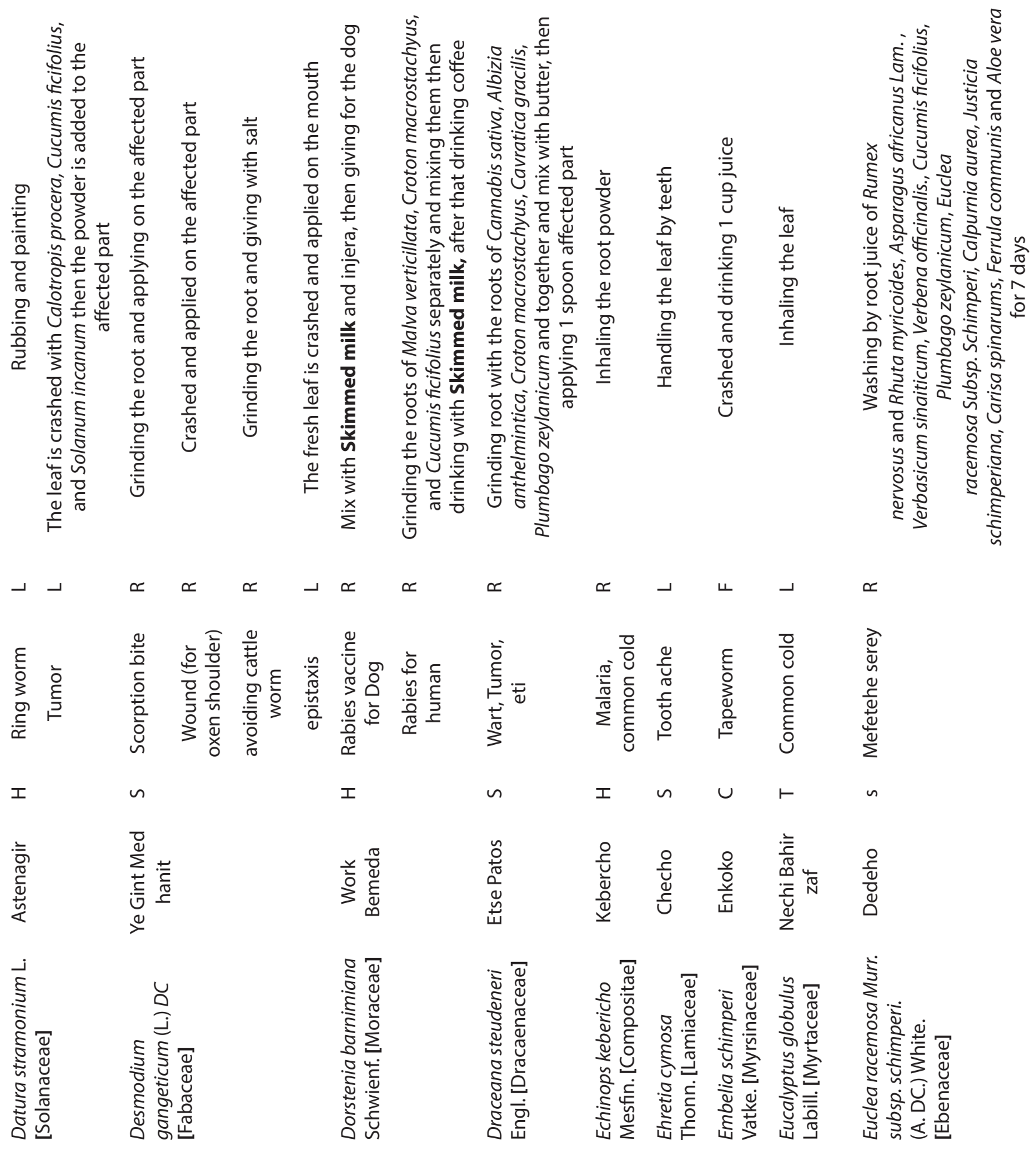


\begin{tabular}{|c|c|c|c|c|c|c|c|c|c|c|}
\hline 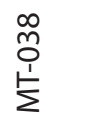 & 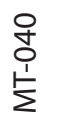 & 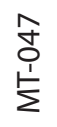 & $\begin{array}{l}\text { nू } \\
\text { ô } \\
\sum \\
\Sigma\end{array}$ & $\begin{array}{l}\overline{\hat{\beta}} \\
\stackrel{1}{1} \\
\Sigma\end{array}$ & $\stackrel{\bar{⺊}}{\Sigma} 8$ & 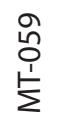 & 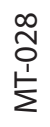 & $\begin{array}{l}\stackrel{+}{m} \\
\stackrel{0}{1} \\
\sum\end{array}$ & $\begin{array}{l}\stackrel{J}{+} \\
\stackrel{1}{1} \\
\sum\end{array}$ & 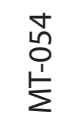 \\
\hline $\begin{array}{l}\infty \\
z^{-} \\
z\end{array}$ & $\underset{\infty}{z}$ & $z$ & $\stackrel{\frac{c}{z}}{z}$ & $\sum_{i=1}^{c}$ & $z_{L^{\prime}} \propto$ & $z$ & 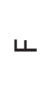 & ч & & $\stackrel{\frac{r}{z}}{z}$ \\
\hline •ே & $\stackrel{\varpi}{\sqcup}$ & 岕 & 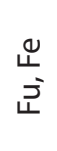 & $\begin{array}{l}0^{\circ} \\
\text { 晏 } \\
\end{array}$ & 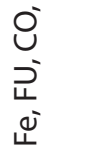 & 운 & 운 & 운 & ' & ' \\
\hline
\end{tabular}
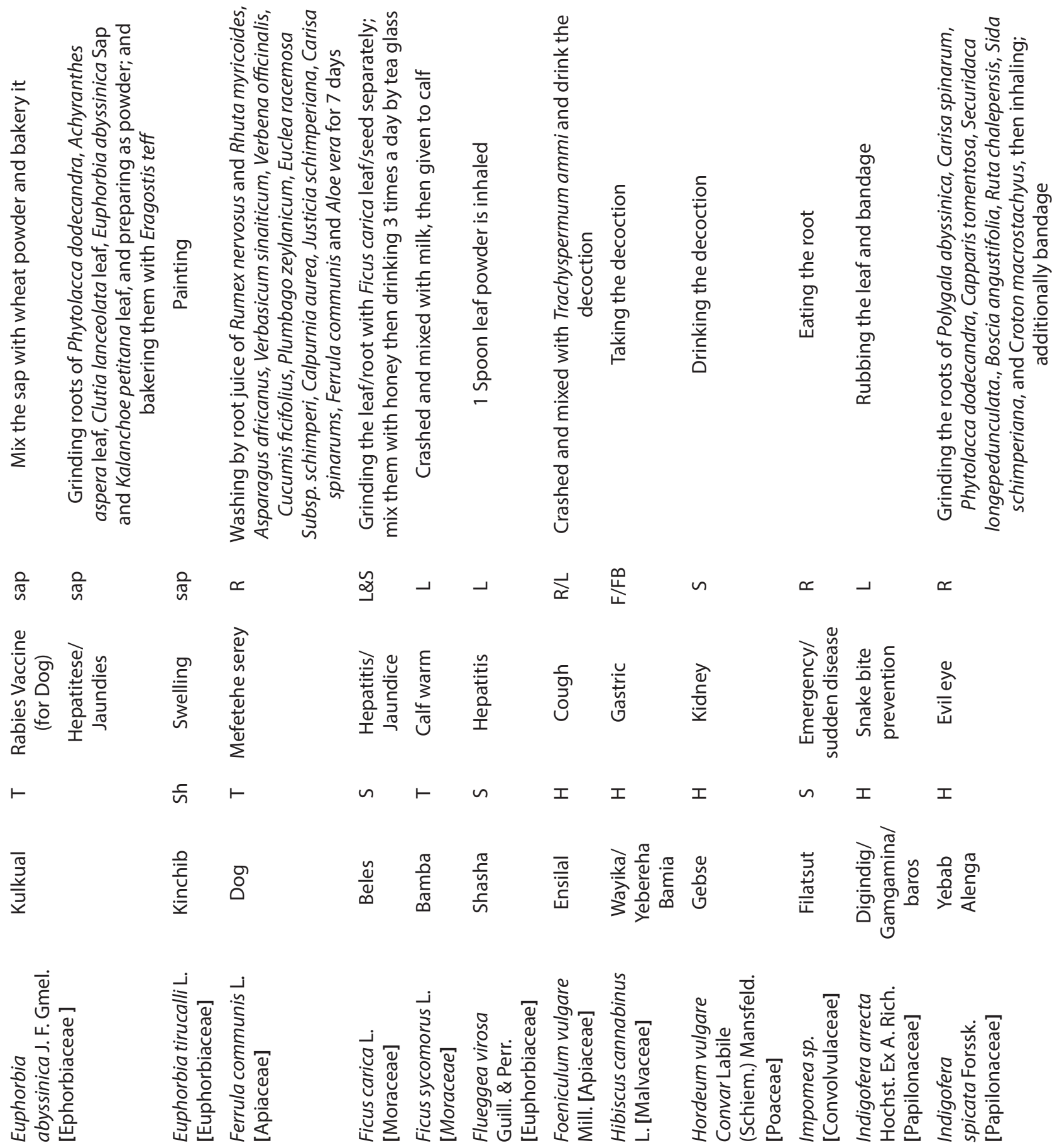


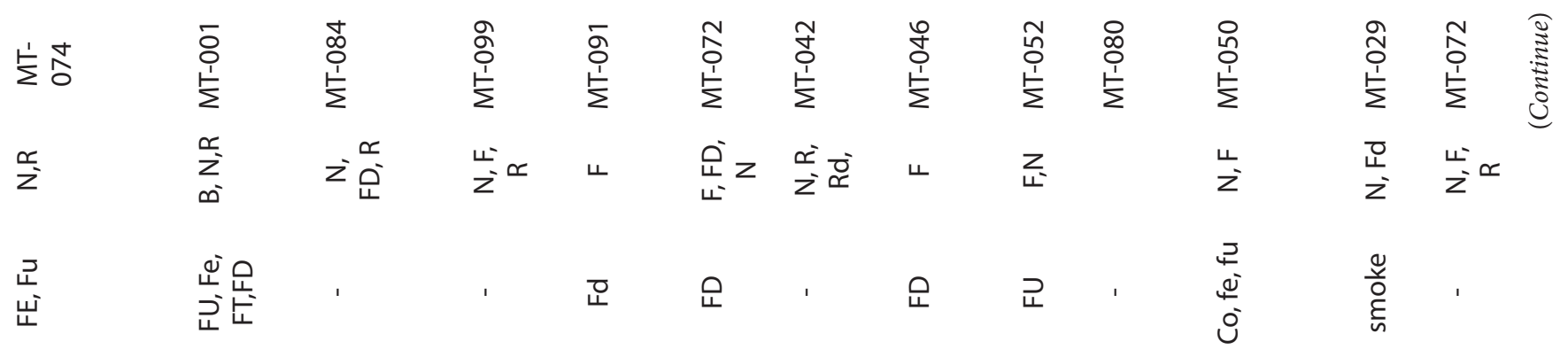
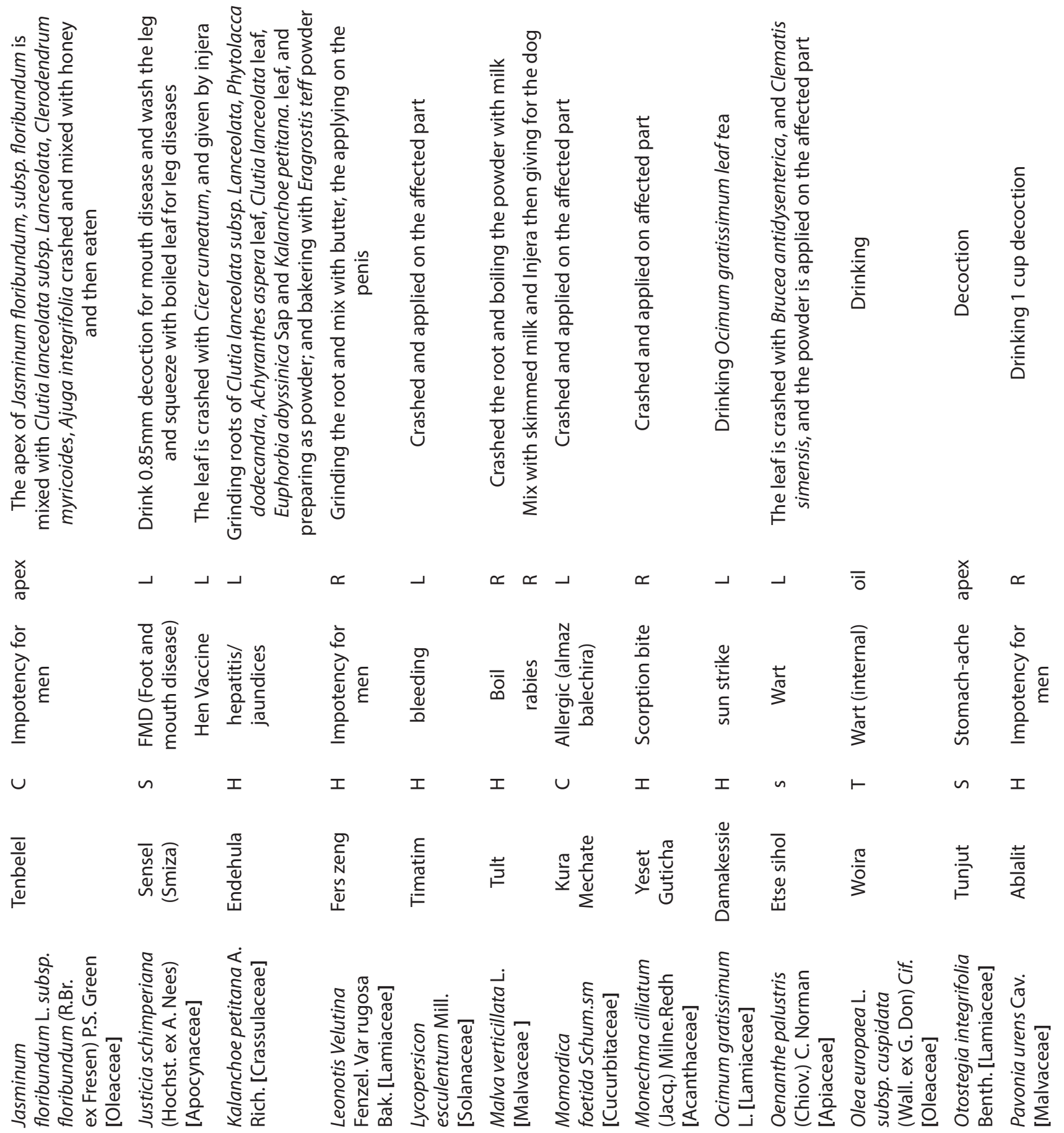


\begin{tabular}{|c|c|c|c|c|c|c|}
\hline $\begin{array}{l}\text { Oे } \\
\text { Oे } \\
\stackrel{1}{\Sigma}\end{array}$ & $\begin{array}{l}\text { ò } \\
\text { ' } \\
\stackrel{1}{\Sigma}\end{array}$ & $\begin{array}{l}\hat{\tilde{o}} \\
\hat{1} \\
\dot{1}\end{array}$ & $\begin{array}{l}\infty \\
\stackrel{\infty}{0} \\
\stackrel{1}{\Sigma} \\
\sum\end{array}$ & $\begin{array}{l}\widetilde{W} \\
\stackrel{1}{1} \\
\stackrel{1}{\Sigma}\end{array}$ & 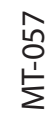 & $\begin{array}{l}\text { पे } \\
\stackrel{1}{2} \\
\stackrel{1}{\Sigma}\end{array}$ \\
\hline I $\stackrel{\frac{r}{z}}{Z}$ & $\begin{array}{l}\frac{r}{\infty} \\
z^{-}\end{array}$ & 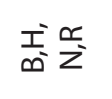 & $\begin{array}{l}L \\
z\end{array}$ & $\underset{\mathbb{L}^{\prime}}{I}$ & & $\begin{array}{l}\stackrel{c}{z} \\
z z\end{array}$ \\
\hline 苀 & 인 & 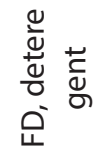 & 呆 & ' & ' & 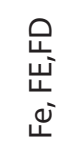 \\
\hline
\end{tabular}
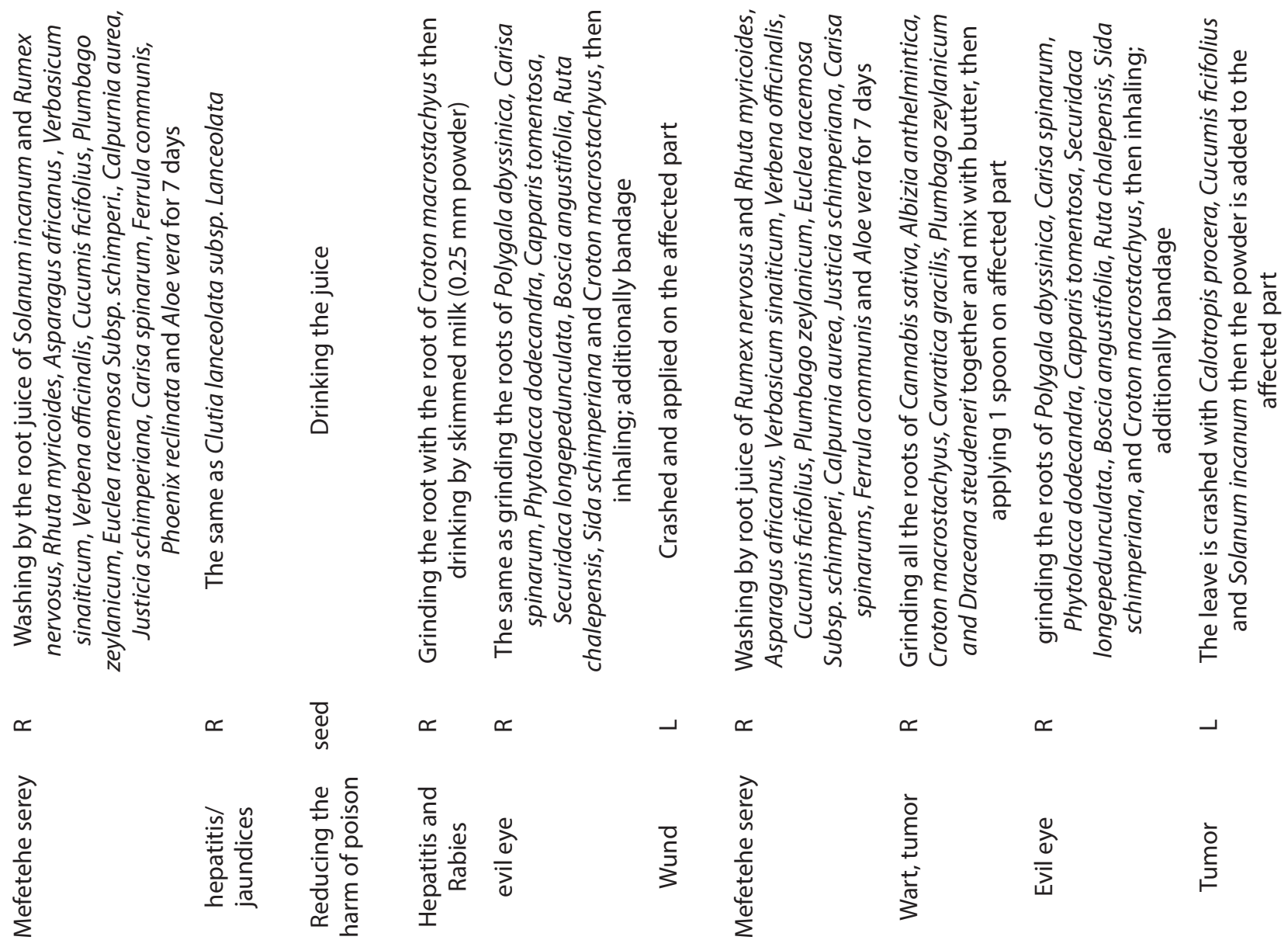

$\propto \quad \propto$
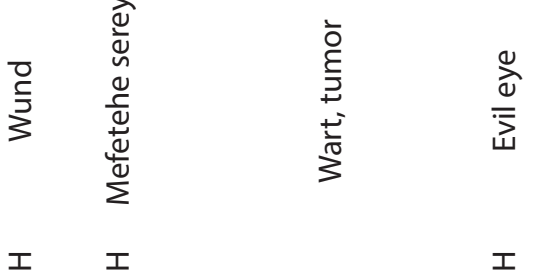

ఏำ

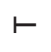

$\sim n$

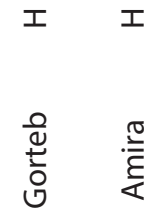

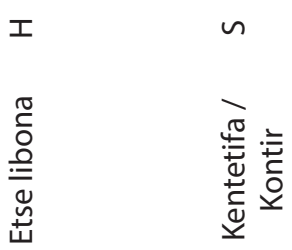

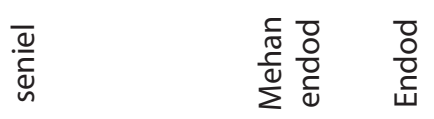
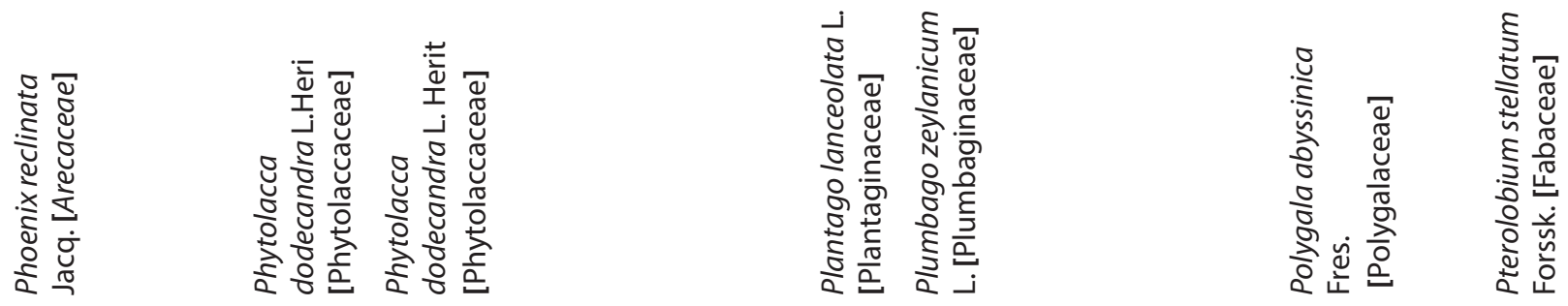


\begin{tabular}{|c|c|c|c|}
\hline $\begin{array}{l}m \\
\mathscr{o} \\
\stackrel{1}{1} \\
\stackrel{1}{\Sigma}\end{array}$ & $\begin{array}{l}\text { P } \\
\text { O } \\
\stackrel{1}{\Sigma}\end{array}$ & $\begin{array}{l}\frac{m}{o} \\
\stackrel{1}{\Sigma}\end{array}$ & $\begin{array}{l}\hat{\sigma} \\
\hat{1} \\
\dot{1}\end{array}$ \\
\hline$\stackrel{\frac{r}{z}}{z}$ & 㞱 & I & 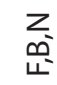 \\
\hline & 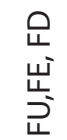 & 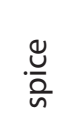 & $\overrightarrow{1}$ \\
\hline
\end{tabular}
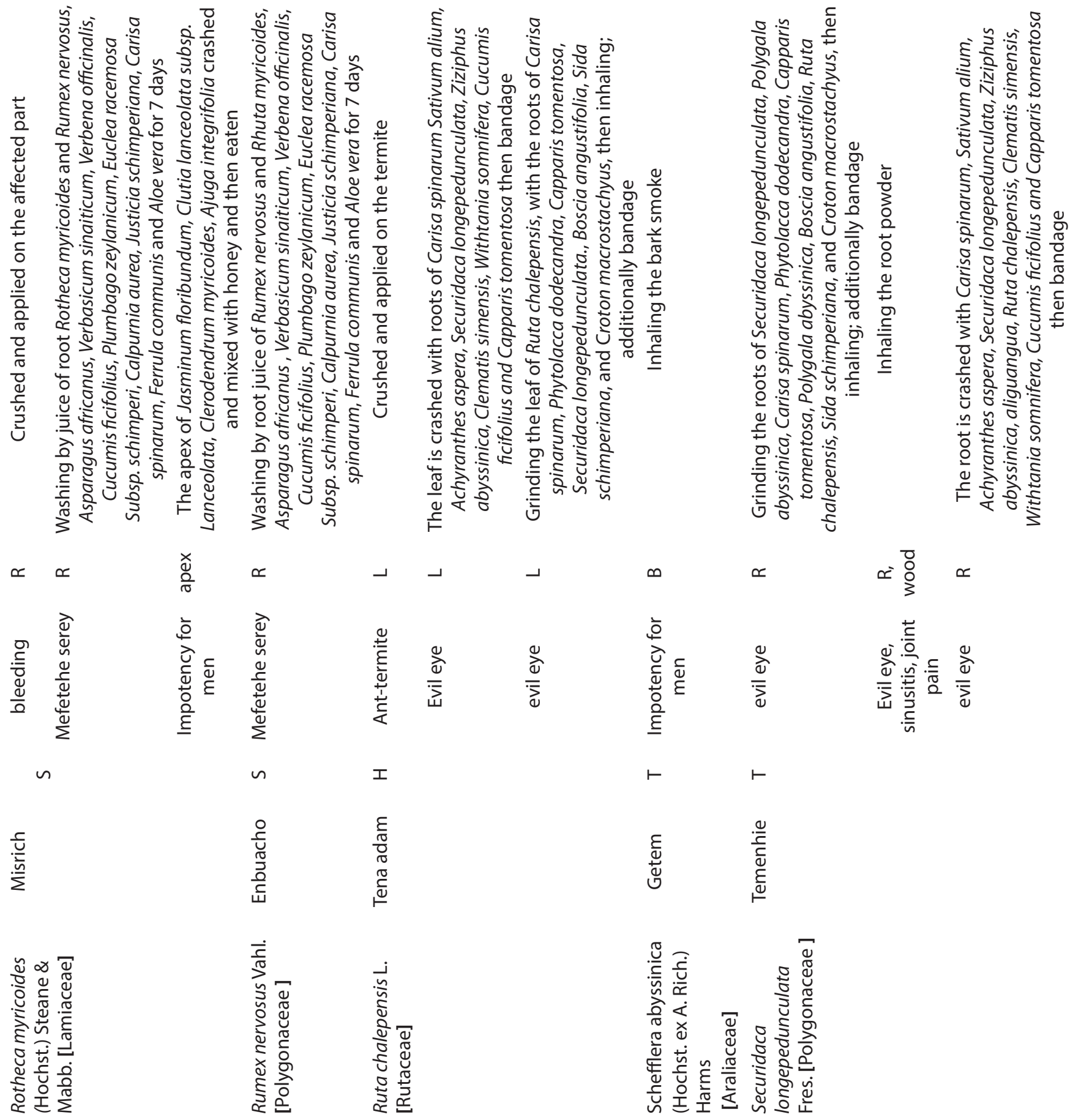


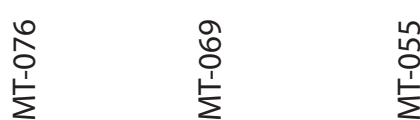

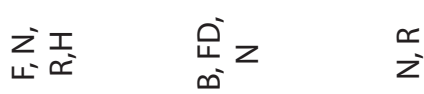

운

它离

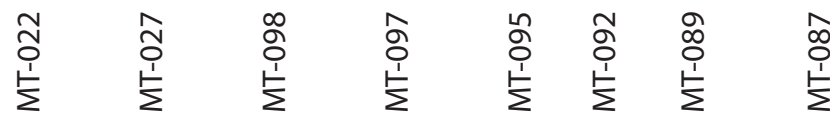

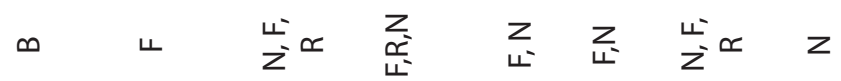

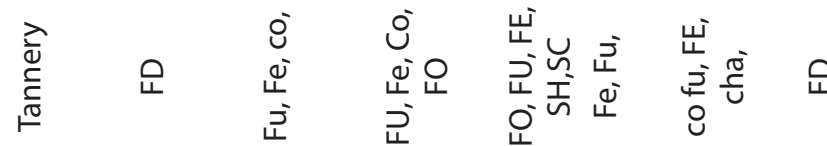

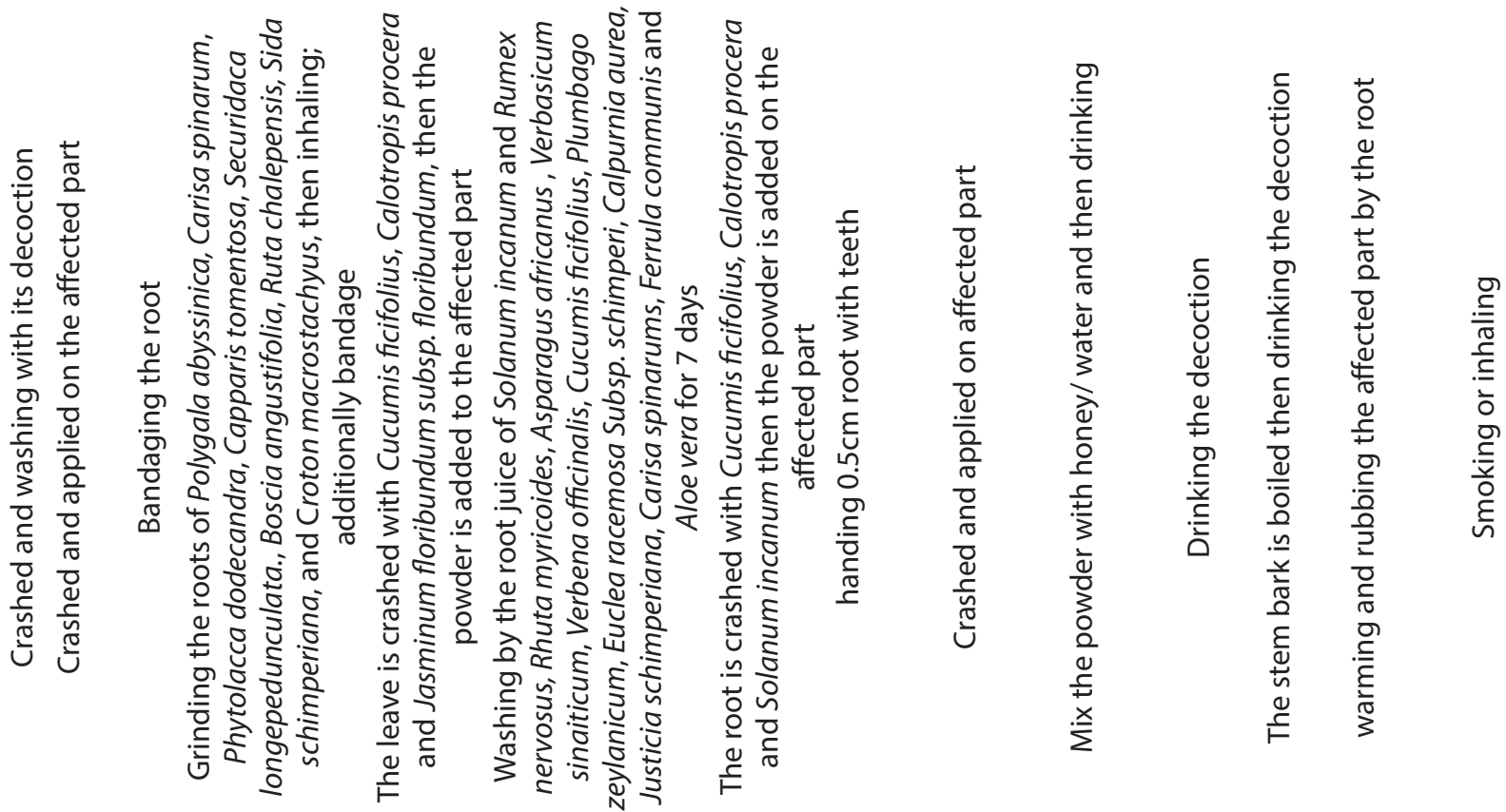

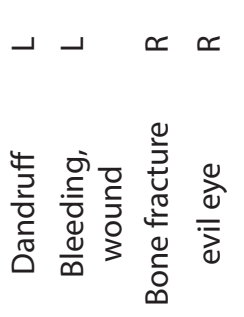

$\sim$

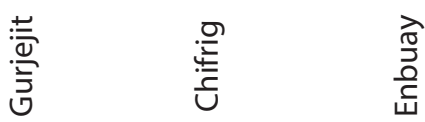

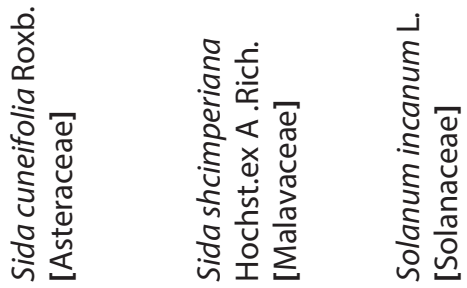

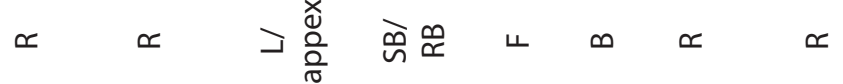

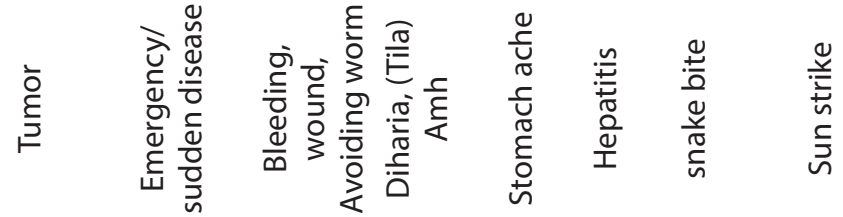

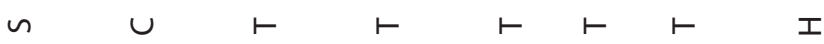

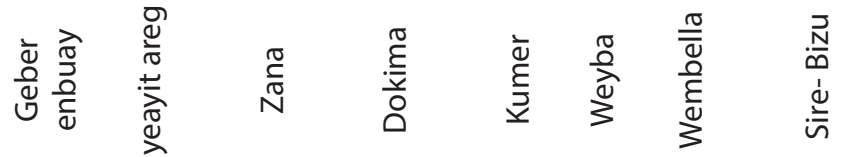

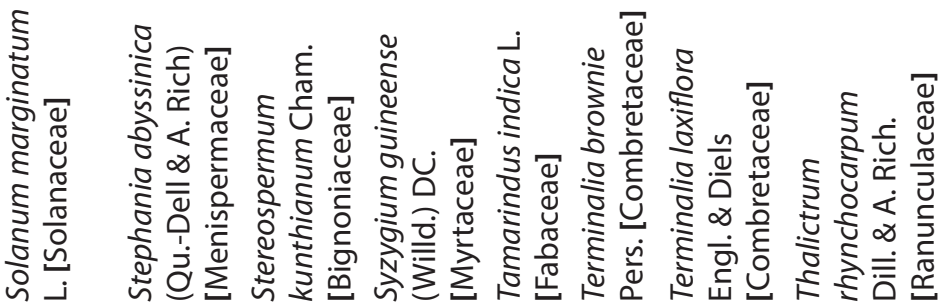


$\stackrel{2}{\infty}$
$\stackrel{0}{1}$
$\stackrel{1}{\Sigma}$

z

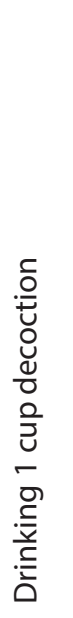

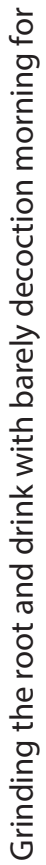

$\propto \propto \propto \propto$

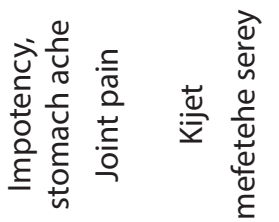

I

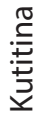

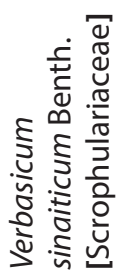

$\stackrel{n}{\infty}$
$\stackrel{\infty}{1}$
$\stackrel{1}{\Sigma}$

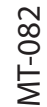

$z_{L}$

군
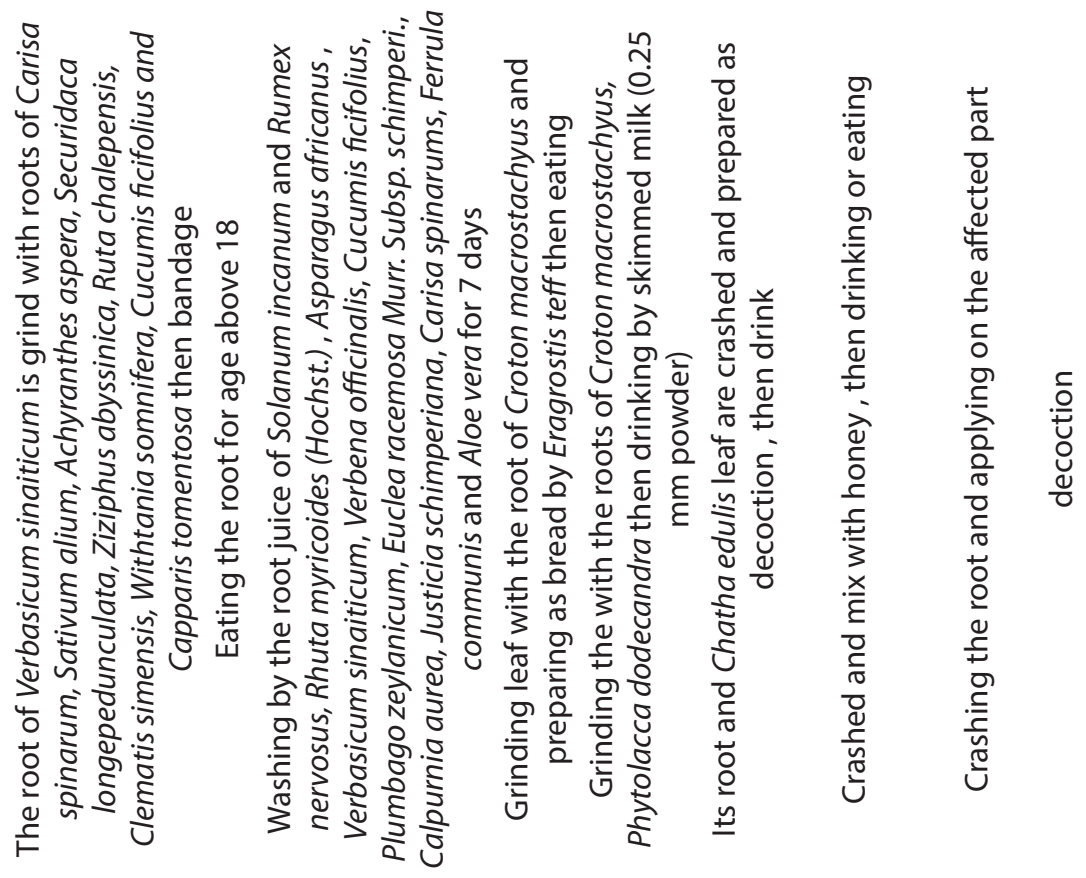

$\simeq \propto$

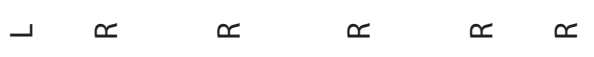

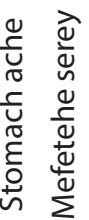
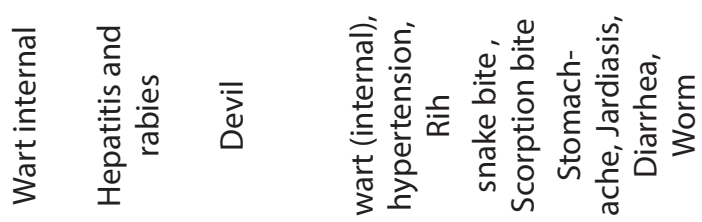

工

喜
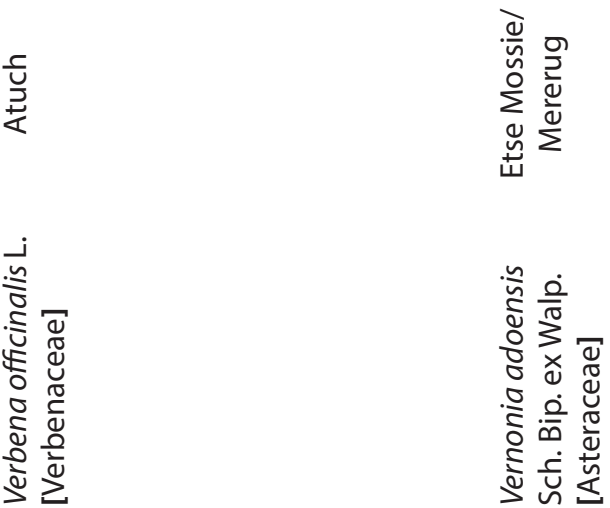

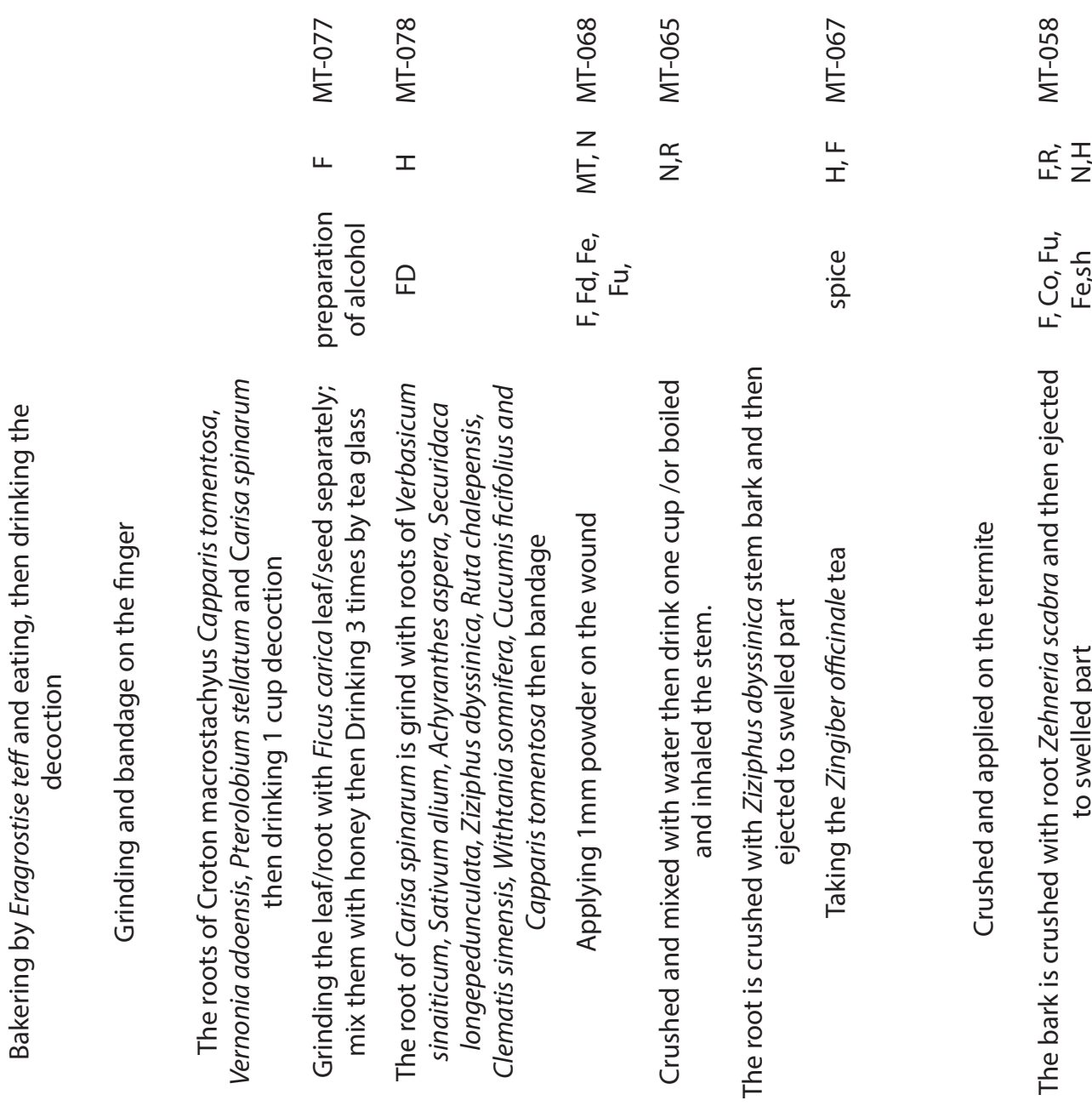

嵀突至

$\frac{\mathscr{0}}{2}$

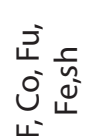

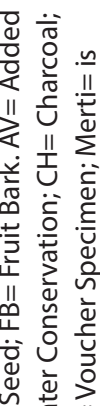
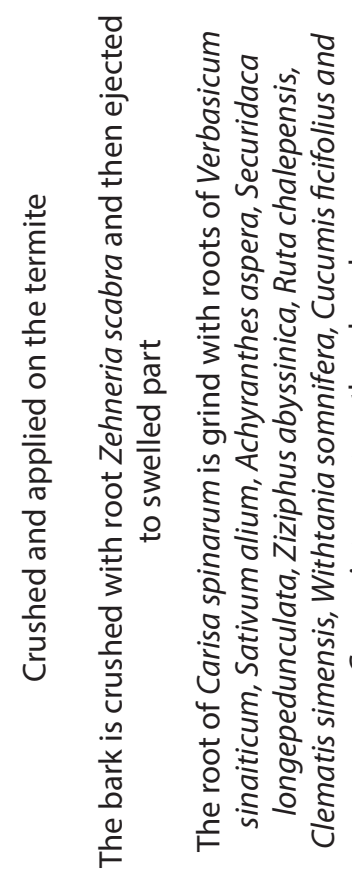

II $\overbrace{}^{\pi}$

芒船

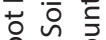

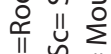

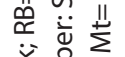

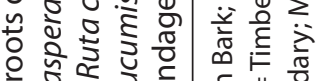

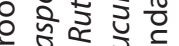

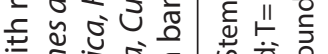

$\mathrm{T}$
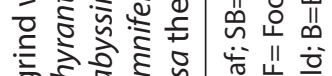

0

可安定

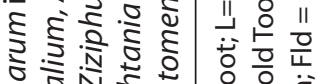

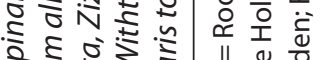

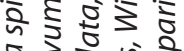

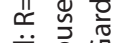

흔

हैं है

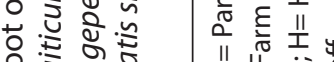

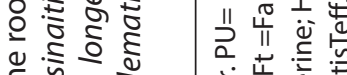

든 들

这芒离:

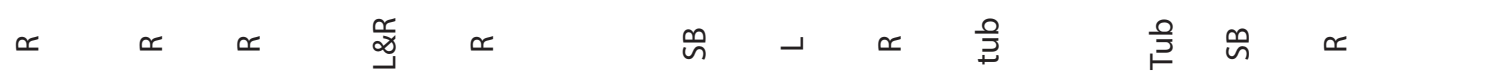

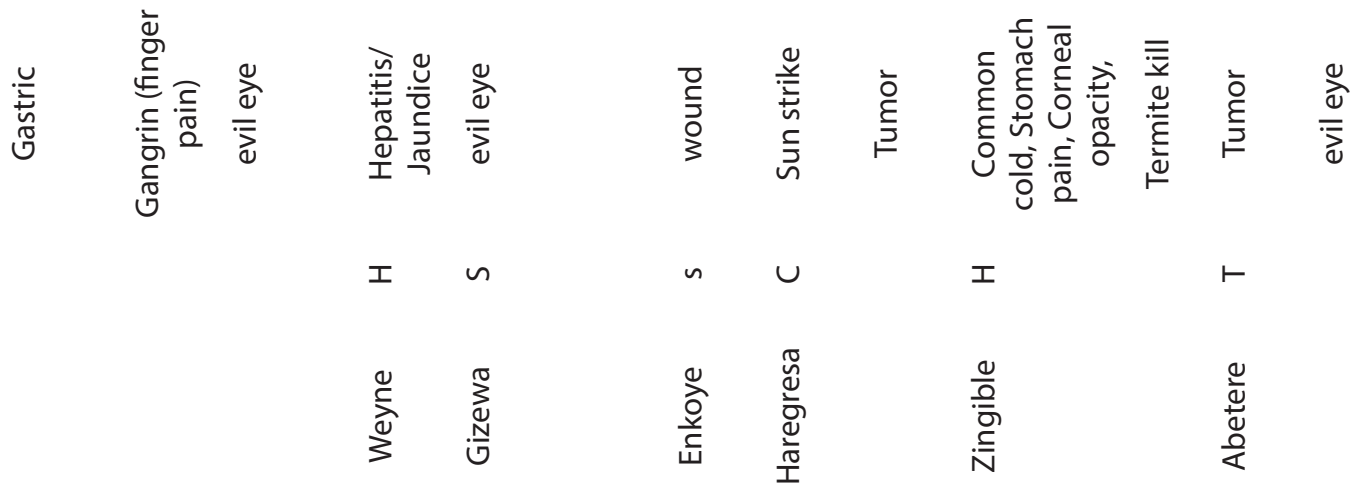

U1

बं

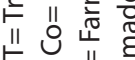

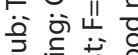

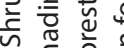

III

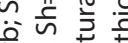

产荌艺常

피 는

过

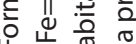

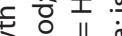

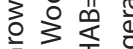

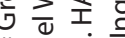

岁岂.

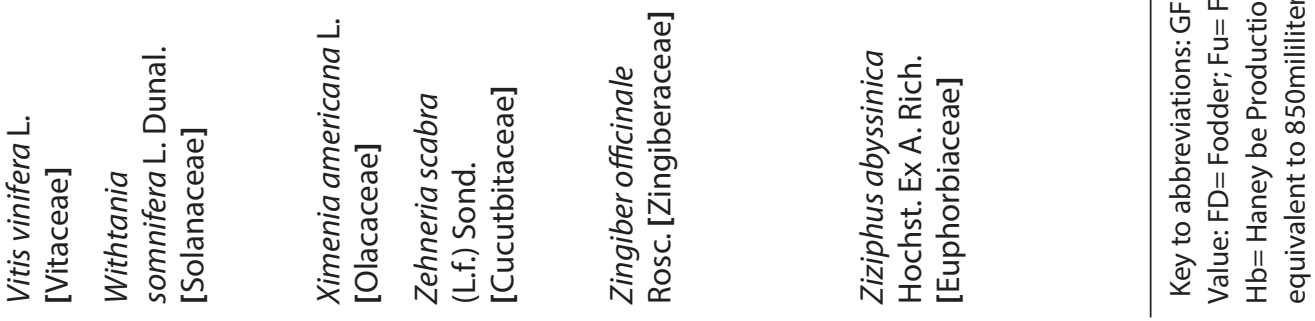


Table 3: Growth forms and number of medicinal plants in the study sites, North western Ethiopia

\begin{tabular}{lccccc}
\hline \multirow{2}{*}{$\begin{array}{l}\text { Growth } \\
\text { Form }\end{array}$} & \multicolumn{5}{c}{ Number of medicinal plants in each study sites } \\
\cline { 2 - 6 } & Walideba & Chalia Debire & Quavier Lomiye & Tenbera & Total \\
\hline Herb & 22 & 19 & 11 & 21 & 36 \\
Shrub & 21 & 16 & 17 & 20 & 30 \\
Tree & 11 & 11 & 10 & 13 & 24 \\
Climber & 7 & 8 & 4 & 4 & 11 \\
Total & 61 & 54 & 42 & 58 & 101 \\
\hline
\end{tabular}

Table 4: Plant parts used in the preparation of therapies in Chilga district, North Western Ethiopia

\begin{tabular}{cc}
\hline Parts Used & Total no. of species \\
\hline Root only & 51 \\
Leaf & 37 \\
Stem Bark & 9 \\
Apex & 6 \\
Seed & 4 \\
Tuber & 3 \\
Fruit & 3 \\
Sap & 3 \\
Oil & 2 \\
Fruit Coat & 1 \\
Root Bark & 1 \\
\hline
\end{tabular}

Table 5: Service categories of medicinal plants in Lowland and midland agroecology of the study area, Northwestern, Ethiopia

\begin{tabular}{lcc}
\hline Use Category & \multicolumn{2}{c}{ Number of species } \\
\cline { 2 - 3 } & Lowland agroecology & Midland agroecology \\
\hline Medicinal use only & 23 & 26 \\
Medicinal \& other Use & 50 & 58 \\
Fodder & 17 & 26 \\
Fuel wood & 28 & 29 \\
Fencing & 24 & 27 \\
Construction & 11 & 8 \\
Charcoal & 2 & 3 \\
Farm and household tools & 8 & 6 \\
Food & 7 & 8 \\
Spice & 2 & 2 \\
Shade & 3 & 3 \\
Timber & 2 & 1 \\
Soil and water conservation & 2 & 2 \\
Others & 7 & 5 \\
\hline
\end{tabular}


Table 6: List of frequently cited medicinal plants and diseases treated in Chilga district, Northwestern Ethiopia (Cited by $\geq 9$ informants)

\begin{tabular}{|c|c|c|c|c|c|c|c|}
\hline $\begin{array}{l}\text { Medicinal plant } \\
\text { (Scientific Name) }\end{array}$ & Ailments Treated & $\begin{array}{l}\text { Parts } \\
\text { Used }\end{array}$ & Walideba & $\begin{array}{l}\text { Chalia } \\
\text { Debire }\end{array}$ & $\begin{array}{l}\text { Quavier } \\
\text { Lomiye }\end{array}$ & Tenbera & Total \\
\hline Zehneria scabra & Sun strike & L & 10 & 7 & 6 & 7 & 30 \\
\hline Carissa spinarum & Evil eye, "meftehe seraye" (Amh) & $\mathrm{R}$ & 5 & 10 & 6 & 6 & 27 \\
\hline Croton macrostachyus & $\begin{array}{l}\text { Evil eye, hepatitis and rabies for } \\
\text { human, wart, tumor, bleeding }\end{array}$ & $R, R B, L, S$ & 1 & 8 & 3 & 4 & 16 \\
\hline Boscia mossambicensis & Evil eye, epilepsy, sinusitis, joint pain & $\mathrm{R}$ & 4 & 1 & 6 & 5 & 16 \\
\hline Achyranthes aspera & Bleeding, hepatitis/jaundice, evil eye & $L, R$ & 6 & 6 & 0 & 2 & 14 \\
\hline Capparis tomentosa & Evil eye, devil & $\mathrm{R}$ & 1 & 5 & 4 & 3 & 13 \\
\hline Desmodium gangeticum & Scorbia & $\mathrm{R}$ & 6 & 3 & 2 & 2 & 13 \\
\hline Vernonia adoensis & Snake bite, diharia & $\mathrm{R}$ & 2 & 6 & 1 & 1 & 10 \\
\hline Bidens pilosa & Bleeding, bone fracture, dandruff & $L, R$ & 4 & 3 & 2 & 1 & 10 \\
\hline Euphorbia abyssinica & Rabies, hepatitis & $S$ & 3 & 4 & 1 & 1 & 9 \\
\hline Allium sativum & Evil eye, giardiasis & TB & 2 & 2 & 1 & 4 & 9 \\
\hline Ruta chalepensis & Evil eye, ant-termite & L & 4 & 1 & 1 & 3 & 9 \\
\hline
\end{tabular}

Note: $\mathrm{L}=$ Leaf; $\mathrm{R}=$ Root; $\mathrm{RB}=$ Root Bark; $\mathrm{S}=\mathrm{Sap} ; \mathrm{TB}=$ Tuber

Table 7: Preference ranking of six medicinal plants used for treating rabies in Chilga district, Northwestern Ethiopia

\begin{tabular}{lccccccccccc}
\hline Plant Species & \multicolumn{1}{c}{ Respondents (R1-R8) } & & Total & Rank \\
\cline { 2 - 12 } & R1 & R2 & R3 & R4 & R5 & R6 & R7 & R8 & & \\
\hline Dorstenia barnimiana & 6 & 6 & 6 & 6 & 6 & 3 & 5 & 5 & 43 & 1 \\
Euphorbia abyssinica & 4 & 4 & 5 & 5 & 5 & 4 & 6 & 6 & 39 & 2 \\
Malva verticillata & 5 & 5 & 4 & 4 & 4 & 2 & 4 & 3 & 31 & 3 \\
Croton macrostachyus & 3 & 1 & 3 & 3 & 2 & 5 & 3 & 4 & 24 & 4 \\
Phytolacca dodecandra & 2 & 2 & 2 & 1 & 3 & 6 & 1 & 2 & 19 & 5 \\
Cucumis ficifolius & 1 & 3 & 1 & 2 & 1 & 1 & 2 & 1 & 12 & 6 \\
\hline
\end{tabular}

Selection and direct matrix ranking of seven multipurpose medicinal plants was also conducted to know the relative importance of these plants to the local community (Table 8). Cordia africana and Syzygium guineense were the first and the second ranked multipurpose species. Of the seven-selected use categories, medicinal and fuel wood were ranked first and second.

\subsection{Health Problems Treated by Medicinal Plants in the Study Area}

Medicinal plants in the study area are used to treat 52 ailments (41 human, 8 livestock and 3 both). Most of medicinal plants $(78.2 \%)$ are used to treat only human disease while $12 \%$ are used to treat only livestock diseases. Of the total underutilized medicinal plants, $15.8 \%$ are used to treat evil eye. Hepatitis and tumor are also treated by 14 plants each (13.9\%). Wart and "Meftehe serey" (Amh) were also treated by 13 plants each. In addition, impotency for men and stomachache were treated by 9 and 8 plants, respectively. According to informants, the majority of underutilized medicinal plants can be used to treat more than one ailment.

Rabies and poultry disease were the most commonly cited livestock diseases which were reported to be treated by seven $(6.9 \%)$ and three $(3.0 \%)$ medicinal plants, respectively. Two medicinal plants treated others for example, calf disease (Yetija beshita), bone 
Table 8: Average direct matrix score of seven medicinal plants and seven use categories in Chilga district, Northwestern Ethiopia

\begin{tabular}{|c|c|c|c|c|c|c|c|c|c|}
\hline Service category & 总 & 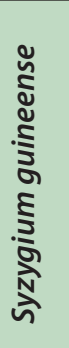 & 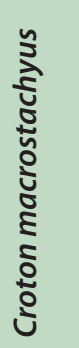 & 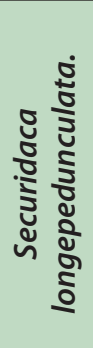 & 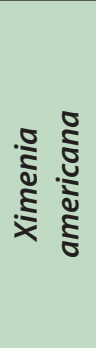 & 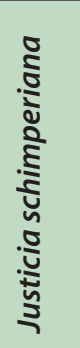 & 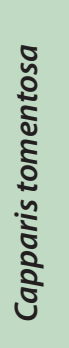 & $\begin{array}{l}\text { Total } \\
\text { Score }\end{array}$ & Rank \\
\hline Medicinal & 3 & 4 & 5 & 5 & 3 & 4 & 4 & 27 & 1 \\
\hline Fuel wood \&Charcoal & 4 & 3 & 4 & 2 & 3 & 4 & 3 & 23 & 2 \\
\hline Fencing & 1 & 2 & 3 & 1 & 2 & 3 & 4 & 16 & 3 \\
\hline Food & 4 & 5 & 0 & 0 & 5 & 0 & 0 & 13 & 4 \\
\hline Construction & 5 & 1 & 1 & 3 & 0 & 0 & 0 & 10 & 5 \\
\hline Farm \& household tools & 3 & 1 & 2 & 1 & 1 & 2 & 0 & 10 & 5 \\
\hline Shade & 2 & 3 & 2 & 3 & 0 & 0 & 0 & 10 & 5 \\
\hline Total score & 20 & 18 & 17 & 15 & 14 & 13 & 11 & & \\
\hline Rank & 1 & 2 & 3 & 4 & 5 & 6 & 7 & & \\
\hline
\end{tabular}

Score criteria: $5=$ best, $4=$ very good, $3=$ good, $2=$ less used, $1=$ least used and $0=$ not used.
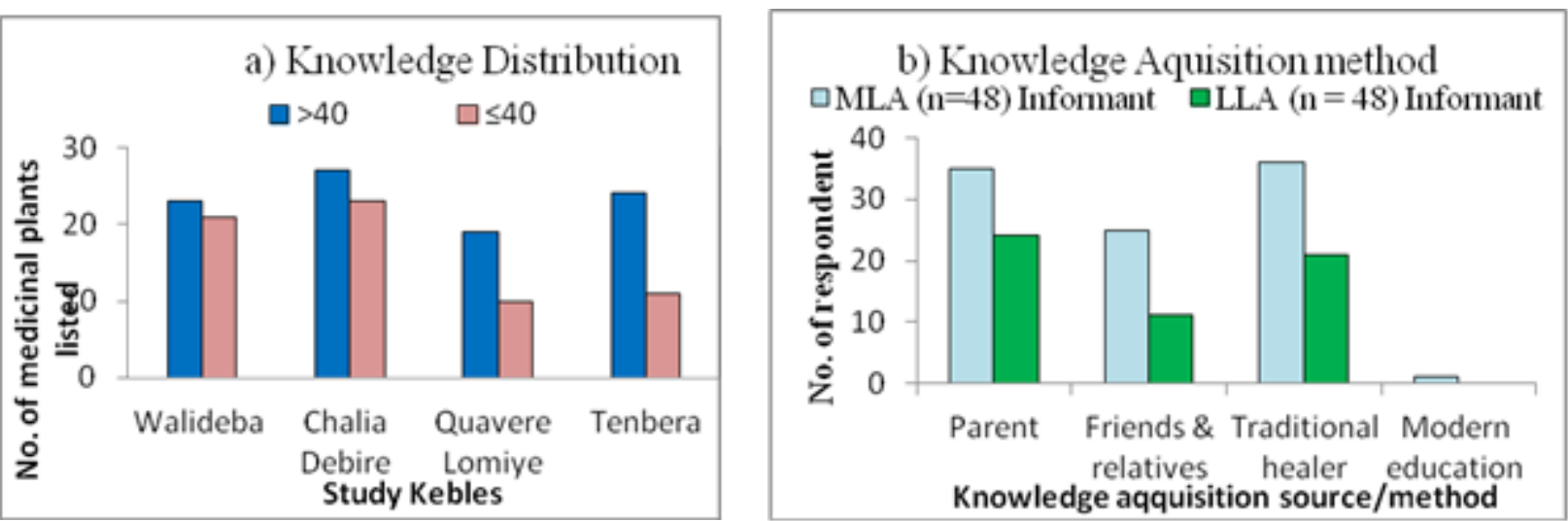

Fig. 2. Knowledge distribution and transfer methods of medicinal plant in Chilga district, Northwestern Ethiopia. MLA=Midland agroecology; LLA= Lowland agroecology

fracture (Tigen / Siberate), emergency/sudden diseases (Abasenga/Qureba) and breast swelling (Yetute ebetete).

\subsection{Medicinal Plant Knowledge Distribution}

The study proved presence of difference in medicinal plant citation among age classes in each study sites (Fig. 2a). Accordingly, elders know better than younger's do. The Spearman's Rank Order Correlation test also confirmed that there was a significant positive correlation between age and medicinal plant list $(\mathrm{r}=0.587, \mathrm{P}<0.01)$. Majority of respondents (73\%) from Midland agroecology and $50 \%$ of respondents from Lowland agroecology indicated that they acquire knowledge from their parent (Fig. 2b). Almost the same proportions of informants got knowledge on medicinal plants from traditional healer. Modern education was the least method of acquisition and transfer in both lowland and midland agroecologies.

The free-list exercise of respondents also shows the presence medicinal plant citation between wealth categories in Quavier Lomiye Kebele (Table 9). Medicinal plant citation of wealthier people were significant ( $\mathrm{p}$ $<0.05)$ than poorest. 


\subsection{Threats of Medicinal Plants}

Plant identification and preference ranking of major medicinal plant threats was conducted based on their destructive effect. Thus, illegal charcoal production (36 total score) and fuel wood collection (31 total score) were the first and second ranked threats (Table 10).

\section{Discussion}

\subsection{Floristic Composition, Diversity and Distribution of Medicinal Plants}

The study area contains high floristic profile of medicinal plant families compared to other semi-arid areas of Ethiopia $^{28,33,35}$. Fabaceae is the most widely used family for medicine purpose. Hailrmariam et al. ${ }^{15}$, Yineger et al. ${ }^{32}$, also report the higher contribution of Fabaceae family in medicinal value.

Of the total medicinal plants (101), 52 medicinal plants were recorded in the field walk plots from the three land uses. Like the present study, the local community elsewhere in Ethiopia collects medicinal plants both from the wild and semi-wild environments ${ }^{13,24,27}$ (Girmaye et al. 2012, Yirga 2009). Ethinobotanical studies elsewhere in Ethiopia such as Lulekal et al. ${ }^{24}$ and Mesfin et al. ${ }^{27}$ in the humid areas, and Adefa ${ }^{1}$, Zenebe et al. ${ }^{35}$, Yirga ${ }^{33,34}$ in semi-arid areas of Ethiopia also documented an appreciable number of medicinal plants.

\subsection{Utilization and Socioeconomic Implication}

Herbaceous plants were the most widely used medicinal plants followed by shrubs and trees in the treatment of ailments in the study sites. The wide utilization of herbs is also reported in other areas of Ethiopia (Adefa ${ }^{1}$ in Tehuledere district, South Wollo, Ethiopia, Amsalu 2010 in Farta district, North Western Ethiopia, Hailemariam et al. ${ }^{15}$ in lowlands of Konta Special Woreda, SNNP of Ethiopia). Contrary to this finding, a greater number of shrubs $^{24}$ (Lulekal et al. in Mana Angetu district, Southeastern Ethiopia, Mesfin ${ }^{27}$ in Wonago district, southern Ethiopia, Teklehaymanot and Giday ${ }^{29}$ in Zegie Peninsula, Northwestern Ethiopia) and trees (Zenebe et al. $^{35}$ in Asgede Tsimbila district, Northern Ethiopia) were recorded. This indicates medicinal plant utilization

Table 9: Mean ( \pm std) of medicinal plants free-list exercise by wealth classes in Chilga district, Northwestern Ethiopia

\begin{tabular}{lcccc}
\hline $\begin{array}{l}\text { Wealth } \\
\text { category }\end{array}$ & $\begin{array}{c}\text { Walideba } \\
(\text { mean } \pm \text { std) }\end{array}$ & $\begin{array}{c}\text { Chalia Debire } \\
(\text { mean } \pm \text { std) }\end{array}$ & $\begin{array}{c}\text { Kuavier Lomiye } \\
(\text { mean } \pm \text { std) }\end{array}$ & $\begin{array}{c}\text { Tenbera Kiwa } \\
(\text { mean } \pm \text { std) }\end{array}$ \\
\hline Rich & $5.38^{\mathrm{a}} \pm 1.18$ & $4.88^{\mathrm{a}} \pm 0.85$ & $3.88^{\mathrm{a}} \pm 0.72$ & $3.13^{\mathrm{a}} \pm 1.22$ \\
Medium & $4.88^{\mathrm{a}} \pm 1.23$ & $4.25^{\mathrm{a}} \pm 0.62$ & $2.88^{\mathrm{ab}} \pm 0.69$ & $4.13^{\mathrm{a}} \pm 1.36$ \\
Poor & $4.63^{\mathrm{a}} \pm 1.59$ & $4.13^{\mathrm{a}} \pm 0.93$ & $1.88^{\mathrm{b}} \pm 0.52$ & $5.50^{\mathrm{a}} \pm 1.67$ \\
Over all mean & $4.96 \pm 0.75$ & $4.42 \pm 0.45$ & $2.88 \pm 0.40$ & $4.25 \pm 0.81$ \\
\hline
\end{tabular}

Means with the same letters ordered vertically are not significant $(P<0.05)$

Table 10: Preference ranking of the major threats for underutilized medicinal plants in Chilga district, Northwestern Ethiopia

\begin{tabular}{|c|c|c|c|c|c|c|c|c|c|c|}
\hline \multirow[t]{2}{*}{ Major Threats } & \multicolumn{8}{|c|}{ Respondents (R1-R8) } & \multirow[t]{2}{*}{ Total } & \multirow[t]{2}{*}{ Rank } \\
\hline & R1 & R2 & R3 & R4 & R5 & R6 & R7 & $\mathrm{R} 8$ & & \\
\hline Illegal Charcoal Production & 5 & 4 & 5 & 5 & 3 & 5 & 4 & 5 & 36 & $1 \mathrm{st}$ \\
\hline Fuel Wood & 3 & 5 & 4 & 4 & 4 & 2 & 5 & 4 & 31 & 2nd \\
\hline Construction & 2 & 3 & 3 & 3 & 4 & 3 & 2 & 3 & 23 & $3 r d$ \\
\hline Agricultural Expansion & 4 & 2 & 1 & 3 & 2 & 4 & 3 & 2 & 21 & 4th \\
\hline Over Grazing/ Free Grazing & 1 & 3 & 1 & 2 & 3 & 3 & 0 & 1 & 14 & 5 th \\
\hline Fire & 0 & 1 & 0 & 1 & 1 & 1 & 1 & 1 & 6 & 6th \\
\hline
\end{tabular}

Ranking criteria: 1-5 and 5-highly destructive, while 1 not destructive 
difference because of variation in culture ${ }^{8}$ agroecologies and topographic features ${ }^{4}$ and easily availability of the species in question ${ }^{5}$.

More than half of plant remedies in the study area were prepared from roots and leaves. Flatie et al. ${ }^{13}$ and Lulekal et al. ${ }^{24}$ also found roots take the highest proportion due to the efficacy of roots in treating the ailments. Conversely, leaves were the most widely used parts of plants ${ }^{1,2,28,32,35}$.

Utilization of a greater number of medicinal plants (101 species) in the study area indicated the strong dependency of the local community on plants to sustain their health care. It also revealed that the endowment and the depth of local people with the associated plant lore. The study conducted in other semi-arid areas of Ethiopia $^{8,28,35}$ (Mesin et al. 2009, Yirga 2009) also proved the dependence of the local community especially rural people on plants to keep up their primary health care. The medicinal plants utilized in this area are reported in other areas of Ethiopia. For instance 22 species by Birhanu ${ }^{6}$ in Gondar Zuria district, Northwestern Ethiopia; 14 species by Giday (2001) by Zay people, Ethiopia; 31 species by Zenebe et al. $^{35}$ in northern Ethiopia;13 species by Ranguatha and Mequanente (2006) in Northwestern Ethiopia and 7 species by Yirga (2010) in Northern Ethiopia. This proves popularity of medicinal plants in treating ailments.

Medicinal plants were utilized for the treatment of both human and livestock ailments. However, the number of diseases treated and plants used for treatment of human ailments were larger. Adafa (2007) and Zenebe et al. ${ }^{35}$ also reported higher number of plants used for treatment of human ailments. Most of the medicinal plants used to treat ailments mixed other plant species. The mixing up of more than one medicinal plant species could attribute to the additive or synergistic effects of the mixtures $^{32}$.

Some medicinal plants were most preferred and commonly used (e.g. Dorstenia barnimiana and Euphorbia abyssinica were used for treatment of rabies) due to their efficacious and low cost (affordable) for the community for specific ailments. Although some plants were available for rabies vaccine they were not popular by most community. Since, the use of the plants was acknowledged only by a few people. Like the present finding other plants e.g Flueggea virosa (Roxb. ex Willd.) Royle (Zenebe et al. ${ }^{35}$ in other areas also used for treatment of rabies virus. Some ailments such as mental problem/ mental disorder (the abnormality of a person mentally or a person unable to do his day to day activities due to illness of the mind), evil spirit (illness of a person due to the force of the devil), Meftehe sery (illness of a person when he given mind distorting plant medicine by other person secretly), evil eye (the evil eye is a specific type of magical curse which is believed to cause harm, illness and even death) and impotency for men were also believed to be cured only by traditional healers using medicinal plants. Similarly, Flatie et al. ${ }^{13}$ and Zenebe et al. ${ }^{35}$ reported "mental problem" believed to cure only by traditional healers and traditional medicines. Medicinal plants were also used for protection and promotion of human physical, spiritual, social, mental and material wellbeing. For instance, traditional healers used medicinal plants to fix bone fracture in the study area. Similarly, Deribe et al. ${ }^{8}$ report the use of medicinal plants for settlement of bone fracture.

Most medicinal plants (67.3 \%) in Chilga district provide multiple uses in addition to their medicinal values (food, construction, fence, fuel wood, farm tools and household implements, fodder, timber or commercial purpose and toothbrush). Other researchers elsewhere in Ethiopia also reported multi-purpose roles of medicinal plants ${ }^{1,21,32,34,35}$. Some medicinal plants such as S. guenees and X. Americana were eaten to supplement the normal food diet. Medicinal plants were also the means of livelihood especially for traditional healers that they fetch income by preparing and selling traditional medicines. Medicinal plants are economically affordable and easily accessible to the rural community and poorest communities. This indicates the contribution of medicinal plants in the livelihood diversification of the local community.

\subsection{Knowledge Distribution and Threats of Medicinal Plant}

The distribution of knowledge varies between different social categories. There is a positive correlation between age and numbers of plants cited i.e. elders cited higher number of medicinal plants than younger people did. Medicinal plant knowledge increases as age increases due to accumulation of knowledge through 
experience $^{1,21,30,35}$ (Giday 2001). In contrary, Yineger and Yewhalaw ${ }^{31}$ reported the loss of such correlation.

The knowledge of medicinal plant transferred from parents, traditional healers and friend to generations. The report of Bekele ${ }^{4}$ and Yirga ${ }^{33}$ is in agreement with the present result that parents, traditional healers and friends are the major sources of knowledge. Mostly it is passed to generations orally and in some cases is secretly. Thus, traditional knowledge of medicinal plants might be lost over time.

Agricultural expansion, over grazing, fire and utilization related factors such as fuel wood and charcoal, construction, house and farm implements are the major threatening factors in both agroecologies. Different researchers have also accounted for these threats ${ }^{4,15,24,27,33}$. Furthermore, most of the plants are collected in the natural environments that are subjected to less management and anthropogenic factors ${ }^{15}$.

\section{Conclusion and Recommendations}

Good numbers of medicinal plants were recorded in lowland and midland agroecologies of the study area. These medicinal plants were claimed by KIs to treat 52 (human and livestock) ailments. Majority of medicinal plant used for treatment of ailments were herbs and shrubs. Most of the encountered medicinal plants provide other services including food, fuel wood and charcoal, construction, timber, farm and household implements, generating income from selling the products and their parts. Medicinal plants were suffering from the threats of agricultural expansion, fuel wood, and construction and over grazing in both agroecologies. Thus, conservation activities from local and national level are needed before the extinction of these plants.

\section{Competing Interest}

The authors declare that they have no competing interests.

\section{Authors' Contributions}

MT: conception and design, data collection and analysis, manuscript writing and final approval of the manuscript.
ZA: data analysis, critical revision and final approval of the manuscript. SZ: critical revision and final approval of the manuscript. All authors read and approved the final manuscript.

\section{Acknowledgement}

We thank to the Development Partnership in higher Education, Department for International development, (DeLPHE) for financial support of this research which otherwise would have faced financial constraints. We are also thankful to the traditional healers and local people of Chilga district who generously shared their knowledge on underutilized medicinal plants. Finally, our acknowledgement passed to the Environmental Protection and Agricultural offices of Chilga district that provide us various basic data and support.

\section{References}

1. Adefa M. 2012. Therapeutic uses of Traditional Medicinal Plants in North Ethiopia: A case study of Tehuledere District, South Wollo, Ethiopia. International Journal of Biomedical Research and Analysis. 3(1):15-22.

2. Amsalu N. An Ethinobotanical Study of Medicinal plants in Farta Woreda, South Gondar Zone of Amhara Region, Ethiopia. [M.Sc Thesis]. Addis Ababa University; 2010. p. 127.

3. Balemie K, Kelbessa E, Asfaw Z. Indigenous medicinal plant utilization, management and threats in Fentalle area, Eastern Shewa, Ethiopia. Ethiopian Journal of Biological Sciences. 2004; 3:37-58.

4. Bekele E. Study on Actual Situation of Medicinal Plants in Ethiopia. 2007. p. 77. Available from http://www. endashaw.com

5. Birhane E, Aynekulu E, Mekuria W, Endale D. Management, use and ecology of medicinal plants in the degraded dry lands of Tigray, Northern Ethiopia. Journal of Horticulture and Forestry. 2011; 3(2):32-41.

6. Birhanu Z. Traditional Use of Medicinal Plants by the Ethnic Groups of Gondar Zuria District, North-western Ethiopia. Journal of Natural Remedies. 2013; 13(1):46-53.

7. CDOA (Chilga District Office of Agricultural), 2012. Chilga District agricultural office 2012 annual report. Aykel, Ethiopia.

8. Deribe K, Amberbir A, Getachew B, Mussema Y. A historical overview of traditional medicine practices 
and policy in Ethiopia. Ethiopian Journal of Health Development. 2006; 20(2):127-34.

9. Edwards S, Tadesse M, Hedberg I. Flora of Ethiopia and Eritrea Vol. 2 Part 2, Canellaceae to Euphorbiaceae. Ethiopia and Upsala, Sweden: The National Herbarium Addis Ababa; 1995. p. 456.

10. Edwards S, Tadesse M, Demisew S, Hedberg I. Flora of Ethiopia and Eritrea Vol. 2 Part 1. Magnoliaceae to Flacourtiaceae. Ethiopia and Upsala, Sweden: The National Herbarium Addis Ababa; 2000. p. 532.

11. Edwards S, Demissew S, Hedberg I. Alliaceae. In: Flora of Ethiopia and Eritrea, Vol. 6. Addis Ababa and Uppsala: The National Herbarium; 1997. p. 586.

12. FAO. Medicinal plants for forest conservation and health care. Non-Wood Forest Products No. 11. Rome; 1997.

13. Flatie T, Gedif T, Asres K, Gebremariam T. Ethnomedical survey of Berta ethnic group Assosa Zone, BenishangulGumuz regional state, mid-west Ethiopia. Journal of Ethnobiology and Ethnomedicine. 2009; 5:14.

14. Gidey M. An Ethinobotanical study of medicinal plants in Zay people in Ethiopia. CBMS Skriftserie. 2001; 3:81-99.

15. Hailemariam T, Demissew S, Asfaw Z. An ethnobotanical study of medicinal plants used by local people in the lowlands of Konta Special Woreda, southern nations, nationalities and peoples regional state, Ethiopia. Journal of Ethnobiology and Ethnomedicine. 2009; 5:26.

16. Hedberg I, Edwards S. Flora of Ethiopia and Eritrea Vol. 3, pittosoraceae to araliaceae. Addis Ababa and Uppsala: The National Herbarium Addis Ababa University; 1989. p. 659.

17. Hedberg I, Kelebessa E, Edwards S, Demissew S, Persson E. Plantaginaceae. In: Flora of Ethiopia and Eritrea, Volume 5. Addis Ababa and Uppsala: The National Herbarium; 2006. p. 690.

18. Hedberg I, Friis I, Edwards S. Asteraceae. In: Flora of Ethiopia and Eritrea Vol. 4, Part 2. Addis Ababa and Uppsala: The National Herbarium; 2004. p. 408.

19. Hedberg I, Friis I, Person E. General Part and Index to Vol 1-7. Flora of Ethiopia and Eritrea Volume 8. Ethiopia and Uppsala, Sweden: The National Herbarium, Addis Ababa; 2009.

20. Hunde D, Njoka J, Asfaw Z, Nyangito M. Wild Edible Fruits of Importance for Human Nutrition in Semiarid Parts of East Shewa Zone, Ethiopia: Associated Indigenous Knowledge and Implications to Food Security. Pakistan Journal of Nutrition. 2011; 10(1):40-50.
21. Hunde D, Asfaw Z, Kelbessa E. Use of Traditional Medicinal Plants by People of 'Boosat' Sub District, Central Eastern Ethiopia. Ethiopian Journal of Health Sci. 2006; 16(2):141-55.

22. Jaenicke H, Hoschele-Zeledon I. Strategic Framework for Underutilized Plant Species Research and Development, with Special Reference to Asia and the Pacific, and to SubSaharan Africa. International Centre for Underutilized Crops, Colombo, Sri Lanka and Global Facilitation Unit for Underutilized Species, Rome, Italy. 2006. p. 33.

23. Kiringe J. Ecological and Anthropological Threats to Ethno-Medicinal Plant Resources and their Utilization in Maasai Communal Ranches in the Amboseli Region of Kenya. Ethnobotany Research and Applications. 2005; 3:231-41.

24. Lulekal E, Kelbessa E, Bekele T, Yineger H. An ethnobotanical study of medicinal plants in Mana Angetu District, southeastern Ethiopia. Journal of Ethnobiology and Ethnomedicine. 2008; 4:10.

25. Magbagbeola J, Adetoso J, Owolabi O. Neglected and underutilized species (NUS): panacea for community focused development to poverty alleviation/poverty reduction in Nigeria. Journal of Economics and International Finance. 2010; 2(10):208-11.

26. Martin G. Ethnobotany: a methods manual. London, UK: Chapman and Hall; 1995.

27. Mesfin FS, Demissew, Teklehaymanot T. An ethnobotanical study of medicinal plants in Wonago Woreda, SNNPR, Ethiopia. Journal of Ethnobiology and Ethnomedicine. 2009; 5:28.

28. Ragunathan M, Mequanente S. Ethionobotanical survey of folk drugs used in Bahir Dar Zuria district, Northwestern Ethiopia. Indian Journal of traditional knowledge. 2009; 8(2):281-4.

29. Teklehaymanot T, Giday M. Ethnobotanical study of medicinal plants used by people in Zegie Peninsula, Northwestern Ethiopia. Journal of Ethnobiology and Ethnomedicine. 2007; 3:12.

30. Tolassa E. Use and Conservation of Traditional Medicinal Plants by Indigenous People in Gimbi Woreda, Western Wellega, Ethiopia. [M.Sc thesis]. Addis Ababa: Addis Ababa University; 2007. p. 121.

31. Yineger $H$, Yewhalaw D. Traditional medicinal plant knowledge and use by local healers in Sekoru District, Jimma Zone, Southwestern Ethiopia. Journal of Ethnobiology and Ethnomedicine. 2007; 3:24. 
32. Yineger H, Yewhalaw D, Teketay D. Ethnomedicinal plant knowledge and practice of the Oromo ethnic group in southwestern Ethiopia. Journal of Ethnobiology and Ethnomedicine. 2008; 4:11.

33. Yirga G. Assessment of indigenous knowledge of medicinal plants in Central Zone of Tigray, Northern Ethiopia. African Journal of Plant Science. 2010a; 4(1):006-11.
34. Yirga G. Ethnobotanical Study of Medicinal Plants in and Around Alamata, Southern Tigray, Northern Ethiopia. Journal of Biological Sciences. 2010b; 2(5):338-44.

35. Zenebe G, Mohammed Z, Zewudie S. An Ethnobotanical Study of Medicinal Plants in Asgede Tsimbila District, Northwestern Tigray, Northern Ethiopia. Ethnobotany Research \& Applications. 2012; 10:305-20. 